Supporting Information

\title{
Methanesulfonyl Iodide
}

\author{
Pradeepa Rajakaruna, John Gorden, and David M. Stanbury* \\ Dept. of Chemistry and Biochemistry \\ Auburn University, Auburn, AL 36849
}

Equilibrium data for formation of $\mathrm{CH}_{3} \mathrm{SO}_{2} \mathrm{I}(\mathrm{aq}) \quad 2$

Kinetic data for hydrolysis of $\mathrm{CH}_{3} \mathrm{SO}_{2} \mathrm{I}(\mathrm{aq})$

Derivation of Eq $12\left(\mathrm{CH}_{3} \mathrm{SO}_{2} \mathrm{I}\right.$ hydrolysis rate law) 3

Crystallographic methods $\quad 4$

Crystal data for $\left(\mathrm{CH}_{3} \mathrm{SO}_{2} \mathrm{I}\right)_{2} \cdot \mathrm{RbI}_{3}$

Crystal data for $\left(\mathrm{CH}_{3} \mathrm{SO}_{2} \mathrm{I}\right)_{4} \cdot \mathrm{KI}_{3} \cdot 2 \mathrm{I}_{2} \quad 14$

Crystal data for $\mathrm{Ca}_{2}\left(\mathrm{CH}_{3} \mathrm{SO}_{2}\right)_{2}\left(\mathrm{CH}_{3} \mathrm{SO}_{3}\right) \mathrm{I}_{3} \cdot\left(\mathrm{H}_{2} \mathrm{O}\right)_{3}$ 


\section{Solution-phase reaction of $\mathrm{I}_{3}{ }^{-}$with MSA.}

Determination of the equilibrium constant for formation of $\mathrm{CH}_{3} \mathrm{SO}_{2} \mathrm{I}$ was performed by mixing solutions of $\mathrm{I}_{3}^{-}$and $\mathrm{CH}_{3} \mathrm{SO}_{2}{ }^{-}$in the stopped-flow instrument and measuring the absorbance at $352 \mathrm{~nm}$ a few ms after the formation of $\mathrm{CH}_{3} \mathrm{SO}_{2} \mathrm{I}$ was complete. These absorbance values were then corrected for a minor contribution from $\mathrm{CH}_{3} \mathrm{SO}_{2} \mathrm{I}$ and then converted to values of $\left[\mathrm{I}_{3}{ }^{-}\right]_{\text {eq }}$ by use of the molar absorptivity if $\mathrm{I}_{3}^{-}$. The correction for $\mathrm{CH}_{3} \mathrm{SO}_{2} \mathrm{I}$ absorbance was performed by the method of successive approximations in fitting the data with eq 7 .

Table S-1: Corrected Values for $\left[\mathrm{I}_{3}^{-}\right]_{\text {eq }}$ in the Equilibrium Reaction between $\mathrm{I}_{3}{ }^{-}$and $\mathrm{CH}_{3} \mathrm{SO}_{2}^{-}$.

\begin{tabular}{|c|c|c|c|}
\hline [MSA], M & {$\left[\mathrm{I}^{-}\right], \mathrm{M}$} & $\mathrm{Abs}_{352}$ & $10^{5}\left[\mathrm{I}_{3}^{-}\right]_{\mathrm{eq}}, \mathrm{M}$ \\
\hline 0.0040 & 0.010 & 0.017 & 0.05 \\
\hline 0.0040 & 0.012 & 0.024 & 0.08 \\
\hline 0.0040 & 0.014 & 0.034 & 0.12 \\
\hline 0.0040 & 0.020 & 0.060 & 0.23 \\
\hline 0.0040 & 0.056 & 0.213 & 0.84 \\
\hline 0.0040 & 0.060 & 0.233 & 0.92 \\
\hline 0.0040 & 0.066 & 0.249 & 0.99 \\
\hline 0.0040 & 0.070 & 0.273 & 1.08 \\
\hline 0.0040 & 0.076 & 0.287 & 1.14 \\
\hline 0.0040 & 0.080 & 0.304 & 1.21 \\
\hline 0.0040 & 0.086 & 0.311 & 1.24 \\
\hline 0.0040 & 0.048 & 0.075 & 0.29 \\
\hline 0.0040 & 0.048 & 0.240 & 0.94 \\
\hline 0.0040 & 0.048 & 0.340 & 1.34 \\
\hline 0.0040 & 0.048 & 0.430 & 1.69 \\
\hline 0.0040 & 0.048 & 0.530 & 2.09 \\
\hline 0.0016 & 0.048 & 0.277 & 1.10 \\
\hline 0.0024 & 0.048 & 0.235 & 0.93 \\
\hline 0.0032 & 0.048 & 0.195 & 0.77 \\
\hline 0.0040 & 0.048 & 0.177 & 0.70 \\
\hline 0.0048 & 0.048 & 0.161 & 0.63 \\
\hline 0.0016 & 0.066 & 0.353 & 1.40 \\
\hline 0.0024 & 0.066 & 0.312 & 1.24 \\
\hline 0.0032 & 0.066 & 0.275 & 1.09 \\
\hline 0.0048 & 0.066 & 0.226 & 0.89 \\
\hline
\end{tabular}

$\left[\mathrm{I}_{2}\right]_{0}=0.02 \mathrm{mM}, \mathrm{T}=(25 \pm 0.5)^{\circ} \mathrm{C}$, un-buffered, $\mu=0.1 \mathrm{M}\left(\mathrm{NaClO}_{4}\right)$. 
Table S-2. Kinetics of $\mathrm{CH}_{3} \mathrm{SO}_{2} \mathrm{I}$ Hydrolysis ${ }^{a}$

\begin{tabular}{lll}
\hline $\mathrm{pH}_{0}$ & {$\left[\mathrm{I}^{-}\right], \mathrm{M}$} & $k_{\mathrm{obs}}, \mathrm{hr}^{-1}$ \\
\hline 2.35 & 0.010 & 0.22 \\
3.53 & 0.010 & 0.035 \\
4.7 & 0.010 & 0.037 \\
6.9 & 0.010 & $0.326^{b}$ \\
6.9 & 0.020 & $0.229^{b}$ \\
6.9 & 0.030 & $0.158^{b}$ \\
6.9 & 0.040 & $0.110^{b}$
\end{tabular}

${ }^{a}[\mathrm{MSA}]=2 \mathrm{mM},\left[\mathrm{I}_{2}\right]_{\mathrm{tot}}=0.2 \mathrm{mM}, \mu=0.1 \mathrm{M}\left(\mathrm{NaClO}_{4}\right) .{ }^{b} 2 \mathrm{mM}$ phosphate buffer.

Derivation of eq 12.

$$
\begin{array}{ll}
\mathrm{CH}_{3} \mathrm{SO}_{2}^{-}+\mathrm{I}_{3}^{-} \rightleftharpoons \mathrm{CH}_{3} \mathrm{SO}_{2} \mathrm{I}+2 \mathrm{I}^{-} & K_{\mathrm{MSI}} \\
\mathrm{CH}_{3} \mathrm{SO}_{2} \mathrm{I}+\mathrm{H}_{2} \mathrm{O} \rightarrow \mathrm{CH}_{3} \mathrm{SO}_{3}{ }^{-}+\mathrm{I}^{-}+2 \mathrm{H}^{+} & k_{\text {hyd }}
\end{array}
$$

$K_{\mathrm{MSI}}=[\mathrm{MSI}]\left[\mathrm{I}^{-}\right]^{2} /\left([\mathrm{MSA}]\left[\mathrm{I}_{3}^{-}\right]\right)$

$\left[\mathrm{I}_{2}\right]_{\text {tot }}=\left[\mathrm{I}_{3}^{-}\right]+[\mathrm{MSI}]$ or $[\mathrm{MSI}]=\left[\mathrm{I}_{2}\right]_{\text {tot }}-\left[\mathrm{I}_{3}^{-}\right]$

so $K_{\mathrm{MSI}}=\left(\left[\mathrm{I}_{2}\right]_{\mathrm{tot}}-\left[\mathrm{I}_{3}^{-}\right]\right)\left[\mathrm{I}^{-}\right]^{2} /\left([\mathrm{MSA}]\left[\mathrm{I}_{3}^{-}\right]\right)$

or $\left[\mathrm{I}_{2}\right]_{\text {tot }}-\left[\mathrm{I}_{3}^{-}\right]=\left[\mathrm{I}_{2}\right]_{\text {tot }} K_{\mathrm{MSI}}[\mathrm{MSA}] /\left(\left[\mathrm{I}^{-}\right]^{2}+K_{\mathrm{MSI}}[\mathrm{MSA}]\right)$

$\mathrm{d}\left[\mathrm{H}^{+}\right] / \mathrm{dt}=2 k_{\text {hyd }}[\mathrm{MSI}]=2 k_{\text {hyd }}\left(\left[\mathrm{I}_{2}\right]_{\text {tot }}-\left[\mathrm{I}_{3}{ }^{-}\right]\right)=-2 \mathrm{~d}\left[\mathrm{I}_{2}\right]_{\text {tot }} / \mathrm{dt}$

or

$\left.\mathrm{d}\left[\mathrm{H}^{+}\right] / \mathrm{dt}\right]=2 k_{\text {hyd }}\left[\mathrm{I}_{2}\right]_{\text {tot }} K_{\mathrm{MSI}}[\mathrm{MSA}] /\left(\left[\mathrm{I}^{-}\right]^{2}+K_{\mathrm{MSI}}[\mathrm{MSA}]\right)$

or

$-\mathrm{d}\left[\mathrm{I}_{2}\right]_{\mathrm{tot}} / \mathrm{dt}=k_{\mathrm{hyd}}\left[\mathrm{I}_{2}\right]_{\mathrm{tot}} K_{\mathrm{MSI}}[\mathrm{MSA}] /\left(\left[\mathrm{I}^{-}\right]^{2}+K_{\mathrm{MSI}}[\mathrm{MSA}]\right)$

so

$k_{\mathrm{obs}}=k_{\mathrm{hyd}} K_{\mathrm{MSI}}[\mathrm{MSA}] /\left(\left[\mathrm{I}^{-}\right]^{2}+K_{\mathrm{MSI}}[\mathrm{MSA}]\right)$ 


\section{X-ray diffraction}

Suitable crystals for X-ray diffraction were isolated using Paratone ${ }^{\mathrm{TM}}$ oil and mounted on a MiTeGen cryo-loop. All data set collection was completed on a Bruker D8 Venture $\kappa$-geometry diffractometer system equipped with a Incoatec $\mathrm{I} \mu \mathrm{S} 3.0$ microfocus sealed tube (Mo $\mathrm{K} \alpha, \lambda=$ $0.71073 \AA$ ) and a multilayer mirror monochromator. The frames were integrated with the Bruker SAINT software package using a narrow-frame algorithm. All data were corrected for absorption effects using the Multi-Scan method (SADABS) and the structures were solved and refined using the Bruker SHELXTL Software Package. ${ }^{1-4}$ Projections were created on Olex2.1. ${ }^{5}$

1. Hubschle, C. B.; Sheldrick, G. M.; Dittrich, B. ShelXle: a Qt graphical user interface for SHELXL. J. Appl. Cryst., 2011, 44, 1281-1284.

2. Krause, L.; Herbst-Irmer, R.; Sheldrick, G. M.; Stalke, D. Comparison of silver and molybdenum microfocus $\mathrm{X}$-ray sources for single-crystal structure determination. $J$. Appl.Cryst., 2015, 48, 3-10.

3. Sheldrick, G. M. SHELXT-Integrated space-group and crystal-structure determination. 2015, Acta Cryst. A71, 3-8.

4. Sheldrick, G. M. Crystal structure refinement with SHELXL. Acta Cryst., 2015, C71, 38.

5. Dolomanov O. V.; Bourhis, L. J.; Gildea, R. J.; Howard, J. A. K.; Puschmann, H. OLEX2: a complete structure solution, refinement and analysis program. J. Appl. Crystallogr., 2009, 42, $339-341$. 


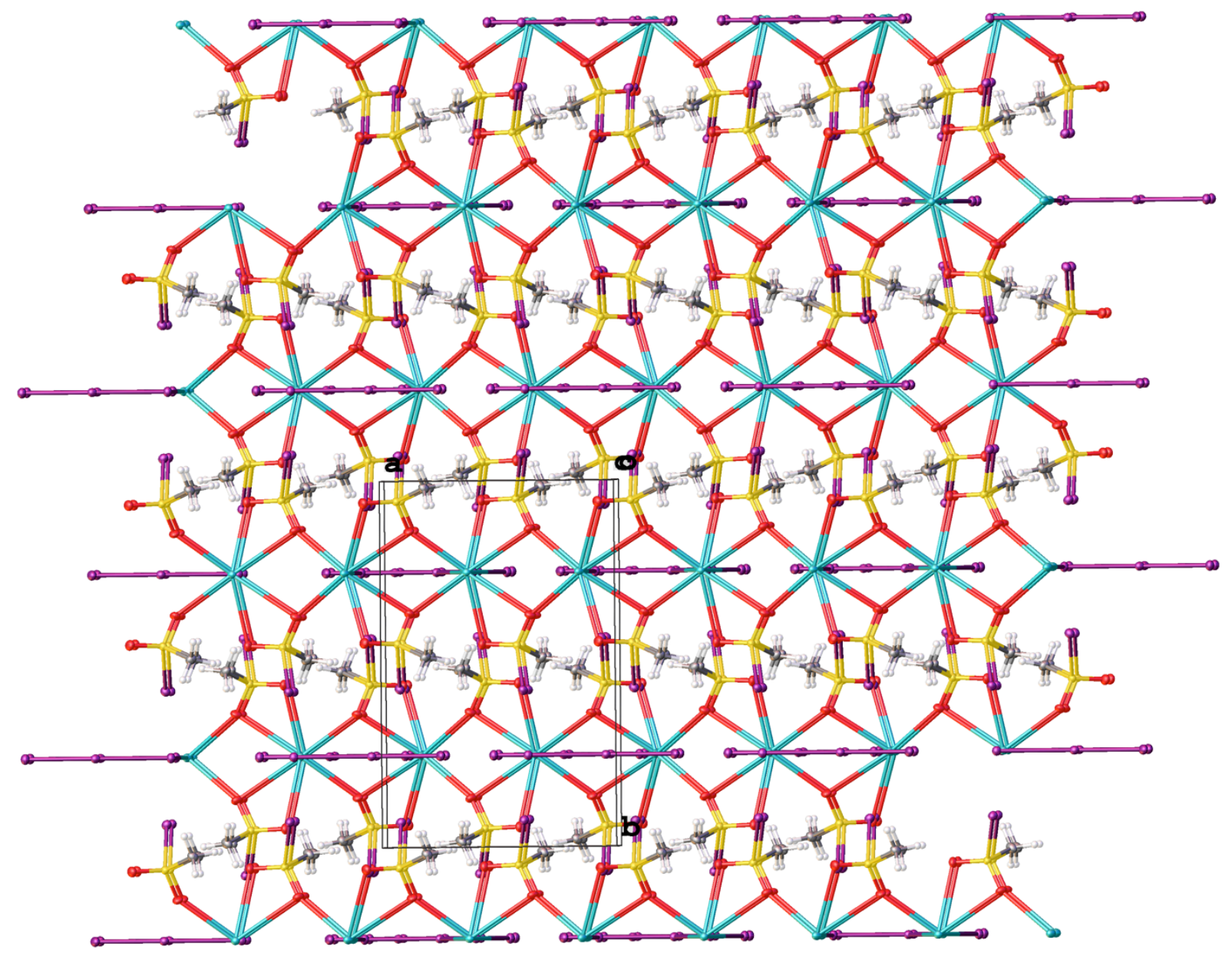

Figure S-1. Molecular packing diagram of $\left(\mathrm{CH}_{3} \mathrm{SO}_{2} \mathrm{I}\right)_{2} \cdot \mathrm{RbI}_{3}$ viewed along the $a b$ plane. 


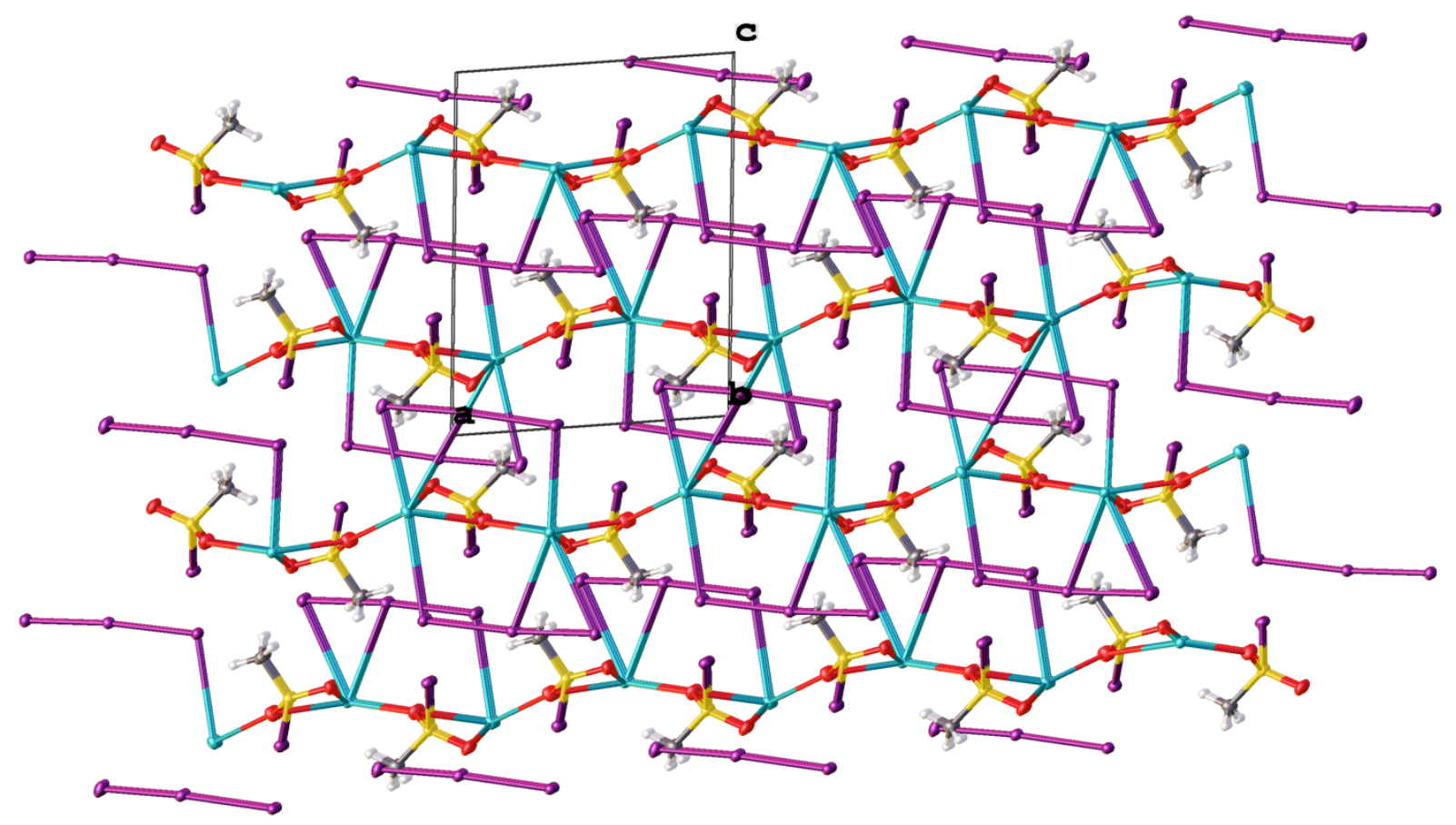

Figure S-2. Molecular packing diagram of $\left(\mathrm{CH}_{3} \mathrm{SO}_{2} \mathrm{I}\right)_{2} \cdot \mathrm{RbI}_{3}$ viewed along the ac plane showing two different coordination modes of $\mathrm{I}_{3}{ }^{-}$with $\mathrm{Rb}$. 


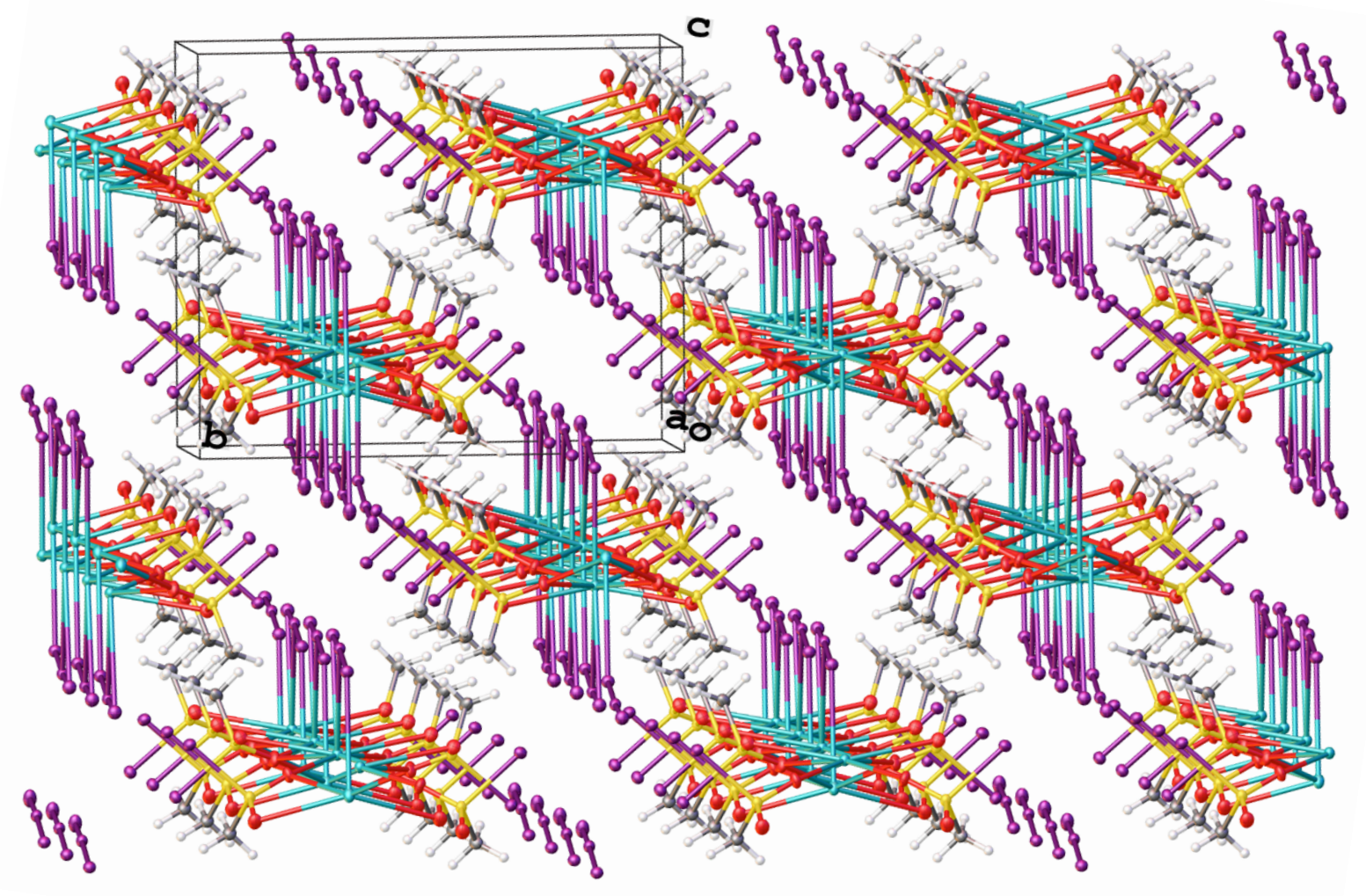

Figure S-3. Molecular packing diagram of $\left(\mathrm{CH}_{3} \mathrm{SO}_{2} \mathrm{I}\right)_{2} \cdot \mathrm{RbI}_{3}$ viewed along the $b c$ plane. 
Table S3. Sample and crystal data for $\left(\mathrm{CH}_{3} \mathrm{SO}_{2} \mathrm{I}\right)_{2} \cdot \mathrm{RbI}_{3}$.

Identification code

Chemical formula

Formula weight

Temperature

Crystal size

Crystal habit

Crystal system

Space group

Unit cell dimensions

Volume

Z

Density (calculated)

Absorption coefficient

$\mathrm{F}(000)$

Diffractometer

Radiation source

Theta range for data collection

Reflections collected

Independent reflections

Absorption correction

Max. and min. transmission

Structure solution technique

Structure solution program

Refinement method

Refinement program

Function minimized

Data / restraints / parameters

Goodness-of-fit on $\mathrm{F}^{2}$

Final $\mathrm{R}$ indices

Largest diff. peak and hole

R.M.S. deviation from mean
DS090518b

$\mathrm{C}_{2} \mathrm{H}_{6} \mathrm{I}_{5} \mathrm{O}_{4} \mathrm{RbS}_{2}$

$878.16 \mathrm{~g} / \mathrm{mol}$

$100(2) \mathrm{K}$

$0.095 \times 0.110 \times 0.215 \mathrm{~mm}$

clear dark brown block

monoclinic

$\mathrm{P} 2{ }_{1} / \mathrm{m}$

$\begin{array}{ll}\mathrm{a}=9.4977(5) \AA & \alpha=90^{\circ} \\ \mathrm{b}=14.7549(8) \AA & \beta=94.818(2)^{\circ} \\ \mathrm{c}=12.3947(7) \AA & \gamma=90^{\circ}\end{array}$

1730.83(16) $\AA^{3}$

4

$3.370 \mathrm{~g} / \mathrm{cm}^{3}$

$12.011 \mathrm{~mm}^{-1}$

1536

Bruker D8 VENTURE $\kappa$-geometry diffractometer

Incoatec $\mathrm{I} \mu \mathrm{S} 3.0$ microfocus sealed tube (Mo K $\alpha, \lambda=$ $0.71073 \AA$ )

2.15 to $36.39^{\circ}$

8638

$8638[\mathrm{R}(\mathrm{int})=0.0241]$

Multi-Scan

0.3950 and 0.1820

direct methods

SHELXT 2014/5 (Sheldrick, 2014)

Full-matrix least-squares on $\mathrm{F}^{2}$

SHELXL-2017/1 (Sheldrick, 2017)

$\Sigma \mathrm{w}\left(\mathrm{F}_{\mathrm{o}}^{2}-\mathrm{F}_{\mathrm{c}}^{2}\right)^{2}$

$8638 / 0 / 142$

1.210

8536 data; $\mathrm{I}>2 \sigma(\mathrm{I}) \quad \mathrm{R} 1=0.0241, \mathrm{wR} 2=0.0681$

all data $\quad \mathrm{R} 1=0.0245, \mathrm{wR} 2=0.0682$

1.365 and $-1.881 \mathrm{e} \AA^{-3}$

$0.213 \mathrm{e}^{-3}$ 
Table S4. Atomic coordinates and equivalent isotropic atomic displacement parameters $\left(\AA^{2}\right)$ for $\left(\mathrm{CH}_{3} \mathrm{SO}_{2} I\right)_{2} \cdot \mathrm{RbI}_{3}$.

$\mathrm{U}(\mathrm{eq})$ is defined as one third of the trace of the orthogonalized $\mathrm{U}_{\mathrm{ij}}$ tensor.

\begin{tabular}{lrrrr} 
& \multicolumn{1}{c}{$\mathrm{x} / \mathrm{a}$} & $\mathrm{y} / \mathrm{b}$ & $\mathrm{z} / \mathrm{c}$ & $\mathrm{U}(\mathrm{eq})$ \\
$\mathrm{I} 1$ & $9269.7(2)$ & $5679.5(2)$ & $6761.9(2)$ & $16.78(4)$ \\
$\mathrm{I} 2$ & $3972.6(2)$ & $5727.8(2)$ & $8217.1(2)$ & $15.97(4)$ \\
$\mathrm{I} 3$ & $15301.2(3)$ & 7500 & $5740.9(2)$ & $17.87(5)$ \\
$\mathrm{I} 4$ & $12305.2(3)$ & 7500 & $5507.4(2)$ & $15.22(4)$ \\
$\mathrm{I} 5$ & $9097.6(3)$ & 7500 & $5036.6(2)$ & $16.09(4)$ \\
$\mathrm{I} 6$ & $3750.7(2)$ & 7500 & $9925.6(2)$ & $15.10(4)$ \\
$\mathrm{I} 7$ & $447.1(3)$ & 7500 & $9377.7(2)$ & $15.33(4)$ \\
$\mathrm{I} 8$ & $-2486.3(3)$ & 7500 & $9037.5(3)$ & $22.36(5)$ \\
$\mathrm{Rb} 1$ & $6379.8(4)$ & 2500 & $7149.9(3)$ & $13.72(6)$ \\
$\mathrm{Rb} 2$ & $1545.2(4)$ & 2500 & $7991.6(3)$ & $17.06(6)$ \\
$\mathrm{S} 1$ & $9330.3(7)$ & $4448.8(4)$ & $8084.4(5)$ & $13.97(10)$ \\
$\mathrm{S} 2$ & $4249.8(7)$ & $4502.7(4)$ & $6925.2(5)$ & $13.85(9)$ \\
O1 & $10730(2)$ & $4407.1(15)$ & $8635(2)$ & $20.7(4)$ \\
O2 & $8809(2)$ & $3626.8(14)$ & $7556.1(18)$ & $19.6(4)$ \\
O3 & $5722(2)$ & $4462.9(15)$ & $6707.8(18)$ & $18.7(3)$ \\
O4 & $3659(3)$ & $3673.8(14)$ & $7317(2)$ & $21.5(4)$ \\
$\mathrm{C} 1$ & $8119(3)$ & $4808(2)$ & $9002(2)$ & $19.3(4)$ \\
$\mathrm{C} 2$ & $3239(3)$ & $4847(2)$ & $5739(2)$ & $21.2(5)$
\end{tabular}

Table S5. Bond lengths $(\AA)$ for $\left(\mathrm{CH}_{3} \mathrm{SO}_{2} \mathrm{I}\right)_{2} \cdot \mathrm{RbI}_{3}$.

$\begin{array}{llllr}\mathrm{I} 1 & \mathrm{~S} 1 & 2.4437(7) & \mathrm{Rb} 1 \mathrm{~S} 2 & 3.5787(7) \\ \mathrm{I} 2 & \mathrm{~S} 2 & 2.4438(7) & \mathrm{Rb} 1 \mathrm{~S} 2^{5} & 3.5787(7) \\ \mathrm{I} 3 & \mathrm{I} 4 & 2.8359(4) & \mathrm{Rb} 1 \mathrm{Rb} 2 & 4.7935(5) \\ \mathrm{I} 3 & \mathrm{Rb}^{1} & 3.7982(5) & \mathrm{Rb} 24^{5} & 2.830(2) \\ \mathrm{I} 4 & \mathrm{I} 5 & 3.0534(4) & \mathrm{Rb} 2 \mathrm{O} 4 & 2.830(2) \\ \mathrm{I} 4 & \mathrm{Rb}^{1} & 3.6193(5) & \mathrm{Rb} 21^{6} & 3.043(2) \\ \mathrm{I} 5 & \mathrm{Rb}^{2} & 3.7518(5) & \mathrm{Rb} 21^{7} & 3.043(2) \\ \mathrm{I} 6 & \mathrm{I} 7 & 3.1541(4) & \mathrm{Rb} 22^{7} & 3.094(2) \\ \mathrm{I} 6 & \mathrm{Rb} 1^{3} & 3.6373(5) & \mathrm{Rb} 22^{6} & 3.094(2) \\ \mathrm{I} 7 & \mathrm{I} 8 & 2.7825(4) & \mathrm{Rb} 21^{7} & 3.5703(7) \\ \mathrm{I} 7 & \mathrm{Rb} 2^{4} & 3.9049(5) & \mathrm{Rb} 21^{6} & 3.5703(7) \\ \mathrm{I} 8 & \mathrm{Rb} 2^{4} & 3.7155(6) & \mathrm{S} 1 \mathrm{O} 1 & 1.444(2) \\ \mathrm{Rb} 1 \mathrm{O} 2 & 2.854(2) & \mathrm{S} 1 \mathrm{O} 2 & 1.446(2) \\ \mathrm{Rb} 1 \mathrm{O} 2^{5} & 2.854(2) & \mathrm{S} 1 \mathrm{C} 1 & 1.765(3) \\ \mathrm{Rb} 1 \mathrm{O} 3 & 3.003(2) & \mathrm{S} 2 \mathrm{O} 4 & 1.447(2) \\ \mathrm{Rb} 1 \mathrm{O} 3^{5} & 3.004(2) & \mathrm{S} 2 \mathrm{O} 3 & 1.447(2)\end{array}$




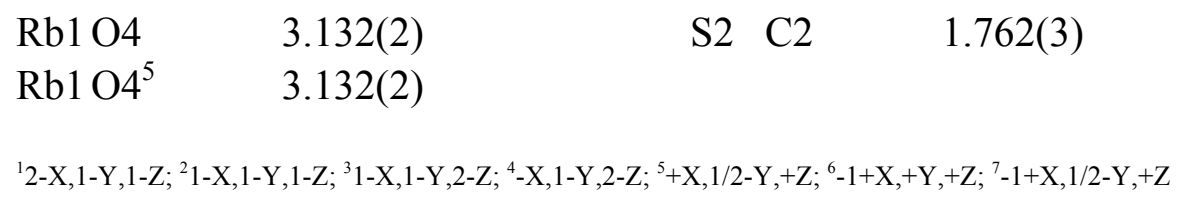

Table S6. Bond angles ( $\left.{ }^{\circ}\right)$ for $\left(\mathrm{CH}_{3} \mathrm{SO}_{2} \mathrm{I}_{2} \cdot \mathrm{RbI}_{3}\right.$.

\begin{tabular}{|c|c|c|c|c|}
\hline & $\mathrm{I} 3 \mathrm{Rb}^{1}{ }^{1}$ & $64.220(9)$ & $\mathrm{O} 1^{7} \mathrm{Rb} 2 \mathrm{O} 2^{7}$ & $47.18(6)$ \\
\hline I3 & I4 I5 & $174.857(11)$ & $\mathrm{O} 4^{5} \mathrm{Rb}_{2} \mathrm{O} 2^{6}$ & $152.07(7)$ \\
\hline I3 & I4 $\mathrm{Rb}^{1}$ & 70.906(9) & $\mathrm{O} 4 \mathrm{Rb}_{2} \mathrm{O}^{6}$ & $103.05(6)$ \\
\hline $\mathrm{I} 5$ & I4 $\mathrm{Rb}^{1}$ & 103.951(10) & $\mathrm{O} 1^{6} \mathrm{Rb} 2 \mathrm{O} 2^{6}$ & $47.18(5)$ \\
\hline I4 & I5 $\mathrm{Rb}^{2}$ & $105.494(10)$ & $\mathrm{O}^{7}{ }^{7} \mathrm{Rb} 2 \mathrm{O}_{2}^{6}$ & $108.31(6)$ \\
\hline I7 & I6 $\mathrm{Rb}^{3}$ & $95.625(9)$ & $\mathrm{O} 2^{7} \mathrm{Rb} 2 \mathrm{O} 2^{6}$ & $65.01(8)$ \\
\hline I8 & I7 I6 & 176.297(12) & $\mathrm{O} 4^{5} \mathrm{Rb}_{2} \mathrm{~S}^{7}$ & $87.20(5)$ \\
\hline I8 & I7 $\mathrm{Rb}^{4}$ & $64.995(11)$ & $\mathrm{O} 4 \mathrm{Rb}_{2} \mathrm{~S}^{7}$ & $159.52(5)$ \\
\hline I6 & I7 $\mathrm{Rb}^{4}$ & $111.302(10)$ & $\mathrm{O} 1^{6} \mathrm{Rb}_{2} \mathrm{~S}_{1}^{7}$ & $124.95(5)$ \\
\hline I7 & I8 $\quad \mathrm{Rb}^{4}$ & $72.263(10)$ & $\mathrm{O}^{7}{ }^{7} \mathrm{Rb} 2 \mathrm{~S}^{7}$ & $23.53(4)$ \\
\hline & $\mathrm{Rb} 1 \mathrm{O} 2^{5}$ & $71.26(9)$ & $\mathrm{O} 2^{7} \mathrm{Rb}_{2} \mathrm{~S} 1^{7}$ & $23.70(4)$ \\
\hline & $\mathrm{Rb} 1 \mathrm{O} 3$ & $67.86(6)$ & $\mathrm{O} 2^{6} \mathrm{Rb}_{2} \mathrm{~S}^{7}{ }^{7}$ & $87.13(4)$ \\
\hline & $\mathrm{Rb} 1 \mathrm{O} 3$ & $138.29(6)$ & $\mathrm{O} 4^{5} \mathrm{Rb}_{2} \mathrm{~S} 1^{6}$ & $159.52(5)$ \\
\hline & $\mathrm{Rb} 1 \mathrm{O}^{5}$ & $138.29(6)$ & $\mathrm{O} 4 \mathrm{Rb} 2 \mathrm{~S}^{6}{ }^{6}$ & $87.20(5)$ \\
\hline & $\mathrm{Rb}^{5} \mathrm{O}^{5}$ & $67.86(6)$ & $\mathrm{O} 1^{6} \mathrm{Rb}_{2} \mathrm{~S} 1^{6}$ & $23.53(4)$ \\
\hline & $\mathrm{Rb} 1 \mathrm{O}^{5}$ & $149.28(9)$ & $\mathrm{O} 1^{7} \mathrm{Rb} 2 \mathrm{~S} 1^{6}$ & $124.95(5)$ \\
\hline & $\mathrm{Rb} 1 \mathrm{O} 4$ & $108.99(6)$ & $\mathrm{O} 2^{7} \mathrm{Rb} 2 \mathrm{~S} 1^{6}$ & $87.13(4)$ \\
\hline & Rb1 O4 & $165.85(6)$ & $\mathrm{O} 2^{6} \mathrm{Rb}_{2} \mathrm{~S} 1^{6}$ & $23.70(4)$ \\
\hline & $\mathrm{Rb} 1 \mathrm{O} 4$ & $47.06(5)$ & $\mathrm{S}^{7}{ }^{\mathrm{Rb}} 2 \mathrm{~S} 1^{6}$ & $107.29(2)$ \\
\hline & $\mathrm{Rb} 1 \mathrm{O} 4$ & $112.66(6)$ & $\mathrm{O} 4^{5} \mathrm{Rb} 2 \mathrm{I} 8^{4}$ & $100.08(5)$ \\
\hline & $\mathrm{Rb} 1 \mathrm{O} 4^{5}$ & $165.85(6)$ & $\mathrm{O} 4 \mathrm{Rb} 2 \mathrm{I}^{4}$ & $100.08(5)$ \\
\hline & $\mathrm{Rb} 1 \mathrm{O}^{5}$ & $108.99(6)$ & $\mathrm{O} 1{ }^{6} \mathrm{Rb} 2 \mathrm{I} 8^{4}$ & 77.61(4) \\
\hline & $\mathrm{Rb} 1 \mathrm{O} 4^{5}$ & $112.66(6)$ & $\mathrm{O}^{7}{ }^{7} \mathrm{Rb} 2 \mathrm{I} 8^{4}$ & 77.61(4) \\
\hline & $\mathrm{Rb}^{\mathrm{O} 4^{5}}$ & $47.06(5)$ & $\mathrm{O} 2^{7} \mathrm{Rb} 2 \mathrm{I} 8^{4}$ & $107.55(4)$ \\
\hline $\mathrm{O} 4$ & $\mathrm{Rb} 1 \mathrm{O} 4^{5}$ & $67.14(8)$ & $\mathrm{O} 2{ }^{6} \mathrm{Rb} 2 \mathrm{I} 8^{4}$ & $107.55(4)$ \\
\hline & $\mathrm{Rb} 1 \mathrm{~S} 2$ & $88.63(5)$ & $\mathrm{S}^{7} \mathrm{Rb}_{2} \mathrm{I}^{4}{ }^{4}$ & $93.524(13)$ \\
\hline & Rb1 S2 & $159.72(5)$ & $\mathrm{S} 1^{6} \mathrm{Rb}_{2} \mathrm{I}^{4}$ & $93.523(13)$ \\
\hline & Rb1 S2 & $23.37(4)$ & $\mathrm{O} 4^{5} \mathrm{Rb}_{2} \mathrm{I}^{2}$ & $76.37(5)$ \\
\hline & $\mathrm{Rb} 1 \mathrm{~S} 2$ & $132.41(4)$ & $\mathrm{O} 4 \mathrm{Rb} 2 \mathrm{I} 5^{2}$ & $76.37(5)$ \\
\hline & $\mathrm{Rb} 1 \mathrm{~S} 2$ & $23.72(4)$ & $\mathrm{O} 1^{6} \mathrm{Rb} 2 \mathrm{I} 5^{2}$ & $103.81(5)$ \\
\hline & $\mathrm{Rb} 1 \mathrm{~S} 2$ & $89.86(4)$ & $\mathrm{O} 1^{7} \mathrm{Rb} 2 \mathrm{I}^{2}$ & $103.81(5)$ \\
\hline & $\mathrm{Rb} 1 \mathrm{~S} 2^{5}$ & $159.72(5)$ & $\mathrm{O} 2^{7} \mathrm{Rb} 2 \mathrm{I} 5^{2}$ & $76.22(4)$ \\
\hline & $\mathrm{Rb} 1 \mathrm{~S} 2^{5}$ & $88.63(5)$ & $\mathrm{O} 2^{6} \mathrm{Rb}^{2} \mathrm{I} 5^{2}$ & $76.22(4)$ \\
\hline $\mathrm{O} 3$ & $\mathrm{Rb} 1 \mathrm{~S} 2^{5}$ & $132.41(4)$ & $\mathrm{S}^{7} \mathrm{Rb}^{2} \mathrm{I}^{2}$ & $89.162(13)$ \\
\hline
\end{tabular}




\begin{tabular}{|c|c|c|c|}
\hline $\mathrm{O} 3^{5} \mathrm{Rb} 1 \mathrm{~S} 2^{5}$ & $23.37(4)$ & $\mathrm{S} 1^{6} \mathrm{Rb} 2 \mathrm{I}^{2}$ & $89.162(13)$ \\
\hline $\mathrm{O} 4 \mathrm{Rb} 1 \mathrm{~S} 2^{5}$ & $89.86(4)$ & $\mathrm{I} 8^{4} \mathrm{Rb} 2 \mathrm{I}^{2}$ & $175.462(13)$ \\
\hline $\mathrm{O} 4^{5} \mathrm{Rb} 1 \mathrm{~S} 2^{5}$ & $23.72(4)$ & $\mathrm{O} 4^{5} \mathrm{Rb} 2 \mathrm{I}^{4}$ & $130.70(5)$ \\
\hline $\mathrm{S} 2 \mathrm{Rb} 1 \mathrm{~S} 2^{5}$ & $111.32(2)$ & $\mathrm{O} 4 \mathrm{Rb} 2 \mathrm{I}^{4}$ & $130.70(5)$ \\
\hline $\mathrm{O} 2 \mathrm{Rb} 1 \mathrm{I}^{1}$ & $79.91(5)$ & $\mathrm{O} 1^{6} \mathrm{Rb} 2 \mathrm{I}^{4}$ & $68.24(5)$ \\
\hline $\mathrm{O} 2^{5} \mathrm{Rb} 1 \mathrm{I} 4^{1}$ & $79.91(5)$ & $\mathrm{O} 1^{7} \mathrm{Rb} 2 \mathrm{I}^{4}$ & $68.24(5)$ \\
\hline $\mathrm{O} 3 \mathrm{Rb} 1 \mathrm{I}^{1}$ & $85.20(4)$ & $\mathrm{O} 2^{7} \mathrm{Rb} 2 \mathrm{I} 7^{4}$ & $71.75(4)$ \\
\hline $\mathrm{O} 3^{5} \mathrm{Rb} 1 \mathrm{I} 4^{1}$ & $85.20(4)$ & $\mathrm{O} 2^{6} \mathrm{Rb} 2 \mathrm{I}^{4}$ & $71.75(4)$ \\
\hline $\mathrm{O} 4 \mathrm{Rb} 1 \mathrm{I}^{1}$ & $114.21(5)$ & $\mathrm{S} 1^{7} \mathrm{Rb} 2 \mathrm{I}^{4}$ & $69.205(12)$ \\
\hline $\mathrm{O} 4^{5} \mathrm{Rb} 1 \mathrm{I} 4^{1}$ & $114.21(5)$ & $\mathrm{S} 1^{6} \mathrm{Rb} 2 \mathrm{I} 7^{4}$ & $69.205(12)$ \\
\hline $\mathrm{S} 2 \mathrm{Rb} 1 \mathrm{I} 4^{1}$ & $99.509(13)$ & $\mathrm{I} 8^{4} \mathrm{Rb} 2 \mathrm{I} 7^{4}$ & $42.743(8)$ \\
\hline $\mathrm{S} 2^{5} \mathrm{Rb} 1 \mathrm{I} 4^{1}$ & $99.509(13)$ & $\mathrm{I}^{2} \mathrm{Rb} 2 \mathrm{I} 7^{4}$ & $141.795(12)$ \\
\hline $\mathrm{O} 2 \mathrm{Rb} 1 \mathrm{I}^{3}$ & $85.35(5)$ & $\mathrm{O} 4^{5} \mathrm{Rb} 2 \mathrm{Rb} 1$ & $38.70(5)$ \\
\hline $\mathrm{O} 2^{5} \mathrm{Rb} 1 \mathrm{I}^{3}$ & $85.35(5)$ & $\mathrm{O} 4 \mathrm{Rb} 2 \mathrm{Rb} 1$ & $38.70(5)$ \\
\hline $\mathrm{O} 3 \mathrm{Rb} 1 \mathrm{I}^{3}$ & $99.08(4)$ & $\mathrm{O} 1^{6} \mathrm{Rb} 2 \mathrm{Rb} 1$ & $109.06(4)$ \\
\hline $\mathrm{O} 3^{5} \mathrm{Rb} 1 \mathrm{I}^{3}$ & $99.08(4)$ & $\mathrm{O} 1^{7} \mathrm{Rb} 2 \mathrm{Rb} 1$ & $109.06(4)$ \\
\hline $\mathrm{O} 4 \mathrm{Rb} 1 \mathrm{I}^{3}$ & $80.60(5)$ & $\mathrm{O} 2^{7} \mathrm{Rb} 2 \mathrm{Rb} 1$ & $140.16(4)$ \\
\hline $\mathrm{O} 4^{5} \mathrm{Rb} 1 \mathrm{I}^{3}$ & $80.60(5)$ & $\mathrm{O} 2{ }^{6} \mathrm{Rb} 2 \mathrm{Rb} 1$ & $140.16(4)$ \\
\hline $\mathrm{S} 2 \mathrm{Rb} 1 \mathrm{I} 6^{3}$ & $90.642(13)$ & $\mathrm{S} 1^{7} \mathrm{Rb} 2 \mathrm{Rb} 1$ & $125.787(11)$ \\
\hline $\mathrm{S} 2^{5} \mathrm{Rb} 1 \mathrm{I} 6^{3}$ & $90.641(12)$ & $\mathrm{S} 1^{6} \mathrm{Rb} 2 \mathrm{Rb} 1$ & $125.787(11)$ \\
\hline $\mathrm{I}^{1} \mathrm{Rb} 1 \mathrm{I}^{3}$ & $161.833(12)$ & $\mathrm{I}^{4} \mathrm{Rb} 2 \mathrm{Rb} 1$ & $93.474(10)$ \\
\hline $\mathrm{O} 2 \mathrm{Rb} 1 \mathrm{I}^{1}$ & $115.84(5)$ & $\mathrm{I}^{2} \mathrm{Rb} 2 \mathrm{Rb} 1$ & $81.988(9)$ \\
\hline $\mathrm{O} 2^{5} \mathrm{Rb} 1 \mathrm{I} 3^{1}$ & $115.84(5)$ & $\mathrm{I}^{4} \mathrm{Rb} 2 \mathrm{Rb} 1$ & $136.217(12)$ \\
\hline $\mathrm{O} 3 \mathrm{Rb} 1 \mathrm{I}^{1}$ & $76.31(4)$ & $\mathrm{O} 1 \mathrm{~S} 1 \mathrm{O} 2$ & $116.43(13)$ \\
\hline $\mathrm{O} 3^{5} \mathrm{Rb} 1 \mathrm{I} 3^{1}$ & $76.31(4)$ & O1 S1 C1 & $109.39(15)$ \\
\hline $\mathrm{O} 4 \mathrm{Rb} 1 \mathrm{I}^{1}$ & $77.22(5)$ & $\mathrm{O} 2 \mathrm{~S} 1 \mathrm{C} 1$ & $109.06(14)$ \\
\hline $\mathrm{O} 4^{5} \mathrm{Rb} 1 \mathrm{I} 3^{1}$ & $77.22(5)$ & O1 S1 I1 & $108.52(10)$ \\
\hline $\mathrm{S} 2 \mathrm{Rb} 1 \mathrm{I} 3^{1}$ & $74.723(12)$ & $\mathrm{O} 2 \mathrm{~S} 1 \mathrm{I} 1$ & $109.37(10)$ \\
\hline $\mathrm{S} 2^{5} \mathrm{Rb} 1 \mathrm{I} 3^{1}$ & $74.724(12)$ & C1 S1 I1 & $103.25(10)$ \\
\hline $\mathrm{I} 4^{1} \mathrm{Rb} 1 \mathrm{I} 3^{1}$ & $44.875(8)$ & O1 S1 Rb2 ${ }^{8}$ & $57.27(9)$ \\
\hline $\mathrm{I}^{3} \mathrm{Rb}^{3} \mathrm{I}^{1}$ & $153.293(12)$ & $\mathrm{O} 2 \mathrm{~S} 1 \mathrm{Rb} 2^{8}$ & $59.34(9)$ \\
\hline $\mathrm{O} 2 \mathrm{Rb} 1 \mathrm{Rb} 2$ & $137.44(4)$ & $\mathrm{C} 1 \mathrm{~S} 1 \mathrm{Rb}^{8}$ & $132.84(10)$ \\
\hline $\mathrm{O} 2^{5} \mathrm{Rb} 1 \mathrm{Rb} 2$ & $137.44(4)$ & I1 $\mathrm{S} 1 \mathrm{Rb}^{8}$ & $123.89(2)$ \\
\hline $\mathrm{O} 3 \mathrm{Rb} 1 \mathrm{Rb} 2$ & $81.45(4)$ & $\mathrm{O} 4 \mathrm{~S} 2 \mathrm{O} 3$ & $115.88(14)$ \\
\hline $\mathrm{O}^{5}{ }^{5} \mathrm{Rb} 1 \mathrm{Rb} 2$ & $81.45(4)$ & $\mathrm{O} 4 \mathrm{~S} 2 \mathrm{C} 2$ & $108.94(15)$ \\
\hline $\mathrm{O} 4 \mathrm{Rb} 1 \mathrm{Rb} 2$ & $34.40(4)$ & O3 S2 C2 & $108.89(15)$ \\
\hline $\mathrm{O} 4^{5} \mathrm{Rb} 1 \mathrm{Rb} 2$ & $34.40(4)$ & $\mathrm{O} 4 \quad \mathrm{~S} 2 \mathrm{I} 2$ & $109.81(10)$ \\
\hline $\mathrm{S} 2 \mathrm{Rb} 1 \mathrm{Rb} 2$ & $58.087(11)$ & O3 $\quad$ S2 $\mathrm{I} 2$ & $108.12(9)$ \\
\hline $\mathrm{S} 2^{5} \mathrm{Rb} 1 \mathrm{Rb} 2$ & $58.088(11)$ & $\mathrm{C} 2 \mathrm{~S} 2 \mathrm{I} 2$ & $104.60(10)$ \\
\hline $\mathrm{I}^{1}{ }^{\mathrm{Rb}} 1 \mathrm{Rb} 2$ & $127.458(11)$ & $\mathrm{O} 4 \mathrm{~S} 2 \mathrm{Rb} 1$ & $60.57(10)$ \\
\hline $\mathrm{I}^{3} \mathrm{Rb}^{2} \mathrm{Rb} 2$ & $70.710(9)$ & $\mathrm{O} 3 \mathrm{~S} 2 \mathrm{Rb} 1$ & $55.41(9)$ \\
\hline $\mathrm{I}^{1}{ }^{1} \mathrm{Rb} 1 \mathrm{Rb} 2$ & $82.583(9)$ & $\mathrm{C} 2 \mathrm{~S} 2 \mathrm{Rb} 1$ & $124.62(10)$ \\
\hline
\end{tabular}




\begin{tabular}{|c|c|c|c|}
\hline $\mathrm{O} 4{ }^{5} \mathrm{Rb} 2 \mathrm{O} 4$ & $75.47(9)$ & $\mathrm{I} 2 \mathrm{~S} 2 \mathrm{Rb} 1$ & $130.62(2)$ \\
\hline $\mathrm{O} 4^{5} \mathrm{Rb} 2 \mathrm{O} 1^{6}$ & $147.76(7)$ & $\mathrm{S} 1 \mathrm{O} 1 \mathrm{Rb} 2^{8}$ & $99.20(11)$ \\
\hline $\mathrm{O} 4 \mathrm{Rb} 2 \mathrm{O} 1^{6}$ & $73.37(7)$ & $\mathrm{S} 1 \mathrm{O} 2 \mathrm{Rb} 1$ & $144.05(13)$ \\
\hline $\mathrm{O} 4^{5} \mathrm{Rb} 2 \mathrm{O} 1^{7}$ & $73.37(7)$ & $\mathrm{S} 1 \mathrm{O} 2 \mathrm{Rb} 2^{8}$ & $96.96(10)$ \\
\hline $\mathrm{O} 4 \mathrm{Rb} 2 \mathrm{O} 1^{7}$ & $147.76(7)$ & $\mathrm{Rb} 1 \mathrm{O} 2 \mathrm{Rb}^{8}$ & $111.87(7)$ \\
\hline $\mathrm{O} 1^{6} \mathrm{Rb} 2 \mathrm{O} 1^{7}$ & $135.29(9)$ & $\mathrm{S} 2 \quad \mathrm{O} 3 \mathrm{Rb} 1$ & $101.22(11)$ \\
\hline $\mathrm{O} 4^{5} \mathrm{Rb} 2 \mathrm{O} 2^{7}$ & $103.05(6)$ & $\mathrm{S} 2 \mathrm{O} 4 \mathrm{Rb} 2$ & $157.14(14)$ \\
\hline $\mathrm{O} 4 \mathrm{Rb} 2 \mathrm{O} 2^{7}$ & $152.07(7)$ & $\mathrm{S} 2 \mathrm{O} 4 \mathrm{Rb} 1$ & $95.71(11)$ \\
\hline $\mathrm{O} 1^{6} \mathrm{Rb} 2 \mathrm{O} 2^{7}$ & $108.31(6)$ & $\mathrm{Rb} 2 \mathrm{O} 4 \mathrm{Rb} 1$ & $106.91(7)$ \\
\hline
\end{tabular}

${ }^{1} 2-X, 1-Y, 1-Z ;{ }^{2} 1-X, 1-Y, 1-Z ;{ }^{3} 1-X, 1-Y, 2-Z ;{ }^{4}-X, 1-Y, 2-Z ;{ }^{5}+X, 1 / 2-Y,+Z ;{ }^{6}-1+X,+Y,+Z ;{ }^{7}-1+X, 1 / 2-Y,+Z ;{ }^{8} 1+X,+Y,+Z$

Table S7. Torsion angles $\left({ }^{\circ}\right)$ for $\left(\mathrm{CH}_{3} \mathrm{SO}_{2} \mathrm{I}\right)_{2} \cdot \mathrm{RbI}_{3}$.

\begin{tabular}{|c|c|c|c|}
\hline A $B \quad C \quad D$ & Angle $/^{\circ}$ & A $B \quad C \quad D$ & Angle $/^{\circ}$ \\
\hline $\mathrm{O} 2 \mathrm{~S} 1 \mathrm{O} 1 \mathrm{Rb} 2^{1}$ & $4.93(16)$ & $\mathrm{O} 4 \mathrm{~S} 2 \mathrm{O} 3 \mathrm{Rb} 1$ & $3.62(16)$ \\
\hline $\mathrm{C} 1 \mathrm{~S} 1 \mathrm{O} 1 \mathrm{Rb} 2^{1}$ & $129.10(12)$ & $\mathrm{C} 2 \mathrm{~S} 2 \mathrm{O} 3 \mathrm{Rb} 1$ & $-119.56(12)$ \\
\hline $\mathrm{S} 1 \mathrm{O} 1 \mathrm{Rb} 2^{1}$ & $-118.93(6)$ & $\mathrm{I} 2 \mathrm{~S} 2 \mathrm{O} 3 \mathrm{Rb} 1$ & $127.34(5)$ \\
\hline $\mathrm{O} 1 \mathrm{~S} 1 \mathrm{O} 2 \mathrm{Rb} 1$ & $139.0(2)$ & $\mathrm{O} 3 \mathrm{~S} 2 \mathrm{O} 4 \mathrm{Rb} 2$ & $-175.1(3)$ \\
\hline $\mathrm{S} 1 \mathrm{O} 2 \mathrm{Rb} 1$ & $14.7(3)$ & $\mathrm{C} 2 \mathrm{~S} 2 \mathrm{O} 4 \mathrm{Rb} 2$ & $-51.9(4)$ \\
\hline $\mathrm{S} 1 \mathrm{O} 2 \mathrm{Rb} 1$ & $-97.6(2)$ & $\mathrm{I} 2 \mathrm{~S} 2 \mathrm{O} 4 \mathrm{Rb} 2$ & $62.1(4)$ \\
\hline $\mathrm{Rb} 2{ }^{1} \mathrm{~S} 1 \mathrm{O} 2 \mathrm{Rb} 1$ & $143.8(3)$ & $\mathrm{Rb} 1 \mathrm{~S} 2 \mathrm{O} 4 \mathrm{Rb} 2$ & $-171.7(4)$ \\
\hline $\mathrm{O} 1 \mathrm{~S} 1 \mathrm{O} 2 \mathrm{Rb} 2^{1}$ & $-4.82(16)$ & O3 S2O4Rb1 & $-3.42(15)$ \\
\hline $\mathrm{S} 1 \mathrm{O} 2 \mathrm{Rb} 2^{1}$ & $-129.16(11)$ & $\mathrm{C} 2 \mathrm{~S} 2 \mathrm{O} 4 \mathrm{Rb} 1$ & $119.74(12)$ \\
\hline $\mathrm{S} 1 \mathrm{O} 2 \mathrm{Rb}^{1}$ & $118.60(5)$ & $\mathrm{I} 2 \mathrm{~S} 2 \mathrm{O} 4 \mathrm{Rb} 1$ & $-126.25(5)$ \\
\hline
\end{tabular}

${ }^{1} 1+\mathrm{X},+\mathrm{Y},+\mathrm{Z}$

Table S8. Anisotropic atomic displacement parameters $\left(\AA^{2}\right)$ for $\left(\mathrm{CH}_{3} \mathrm{SO}_{2} \mathrm{I}\right)_{2} \cdot \mathrm{RbI}_{3}$.

The anisotropic atomic displacement factor exponent takes the form: $-2 \pi^{2}\left[\mathrm{~h}^{2} \mathrm{a}^{* 2} \mathrm{U}_{11}+\ldots+2 \mathrm{~h} \mathrm{k} \mathrm{a}{ }^{*} \mathrm{~b}^{*} \mathrm{U}_{12}\right]$

\begin{tabular}{lrrrrrr} 
Atom & \multicolumn{1}{c}{$\mathrm{U}_{11}$} & \multicolumn{1}{c}{$\mathrm{U}_{22}$} & \multicolumn{1}{c}{$\mathrm{U}_{33}$} & \multicolumn{1}{c}{$\mathrm{U}_{12}$} & $\mathrm{U}_{13}$ & \multicolumn{1}{c}{$\mathrm{U}_{23}$} \\
$\mathrm{I} 1$ & $17.74(7)$ & $17.18(7)$ & $15.19(7)$ & $-1.83(5)$ & $0.10(5)$ & $1.25(5)$ \\
$\mathrm{I} 2$ & $15.70(7)$ & $15.55(6)$ & $16.84(7)$ & $-0.88(5)$ & $2.39(5)$ & $-1.75(5)$ \\
$\mathrm{I} 3$ & $14.18(9)$ & $19.15(10)$ & $20.17(10)$ & 0 & $0.81(8)$ & 0 \\
$\mathrm{I} 4$ & $16.19(9)$ & $13.84(8)$ & $15.94(9)$ & 0 & $3.16(7)$ & 0 \\
$\mathrm{I} 5$ & $13.63(9)$ & $18.06(9)$ & $16.46(9)$ & 0 & $0.58(7)$ & 0 \\
$\mathrm{I} 6$ & $14.78(9)$ & $16.29(9)$ & $14.33(9)$ & 0 & $1.76(7)$ & 0 \\
$\mathrm{I} 7$ & $16.51(9)$ & $13.33(8)$ & $15.87(9)$ & 0 & $-0.28(7)$ & 0 \\
$\mathrm{I} 8$ & $15.71(10)$ & $17.69(10)$ & $32.44(13)$ & 0 & $-5.24(9)$ & 0 \\
Rb1 & $14.03(13)$ & $12.45(12)$ & $14.47(13)$ & 0 & $-0.01(10)$ & 0 \\
Rb2 & $16.56(14)$ & $13.55(13)$ & $21.90(15)$ & 0 & $6.48(12)$ & 0
\end{tabular}




$\begin{array}{lrrrrrr}\text { S1 } & 12.6(2) & 12.5(2) & 16.5(2) & -0.64(18) & -0.66(18) & -0.56(18) \\ \text { S2 } & 12.6(2) & 12.0(2) & 16.8(2) & -0.39(18) & 1.12(18) & -0.03(18) \\ \text { O1 } & 16.1(8) & 19.8(8) & 25.0(10) & 1.1(7) & -5.6(7) & 0.5(7) \\ \text { O2 } & 19.9(8) & 12.9(7) & 25.7(10) & -2.4(7) & 0.0(7) & -5.3(7) \\ \text { O3 } & 12.9(7) & 20.8(8) & 22.8(9) & 0.6(7) & 4.0(7) & -1.5(7) \\ \text { O4 } & 24.0(9) & 14.0(8) & 27.4(10) & -3.7(7) & 7.2(8) & 1.6(7) \\ \text { C1 } & 20.8(11) & 18.9(10) & 18.5(10) & 2.7(9) & 3.7(9) & 0.0(9) \\ \text { C2 } & 22.4(12) & 20.1(11) & 20.0(11) & 4.6(9) & -4.1(9) & -3.2(9)\end{array}$

Table S9. Hydrogen atomic coordinates and isotropic atomic displacement parameters $\left(\AA^{2}\right)$ for $\left(\mathrm{CH}_{3} \mathrm{SO}_{2} \mathrm{I}\right)_{2} \cdot \mathrm{RbI}_{3}$.

\begin{tabular}{lrlll} 
Atom & \multicolumn{1}{l}{$x$} & \multicolumn{1}{l}{$y$} & \multicolumn{1}{l}{ U } & $\mathrm{U}(\mathrm{eq})$ \\
H1A & 8014.84 & 4332.15 & 9541.17 & 29 \\
H1B & 7200.7 & 4927.79 & 8606.83 & 29 \\
H1C & 8469.97 & 5362.61 & 9366.65 & 29 \\
H2A & 3610.57 & 5420.66 & 5482.77 & 32 \\
H2B & 2252.49 & 4930 & 5895.93 & 32 \\
H2C & 3290.11 & 4382.68 & 5178.61 & 32
\end{tabular}




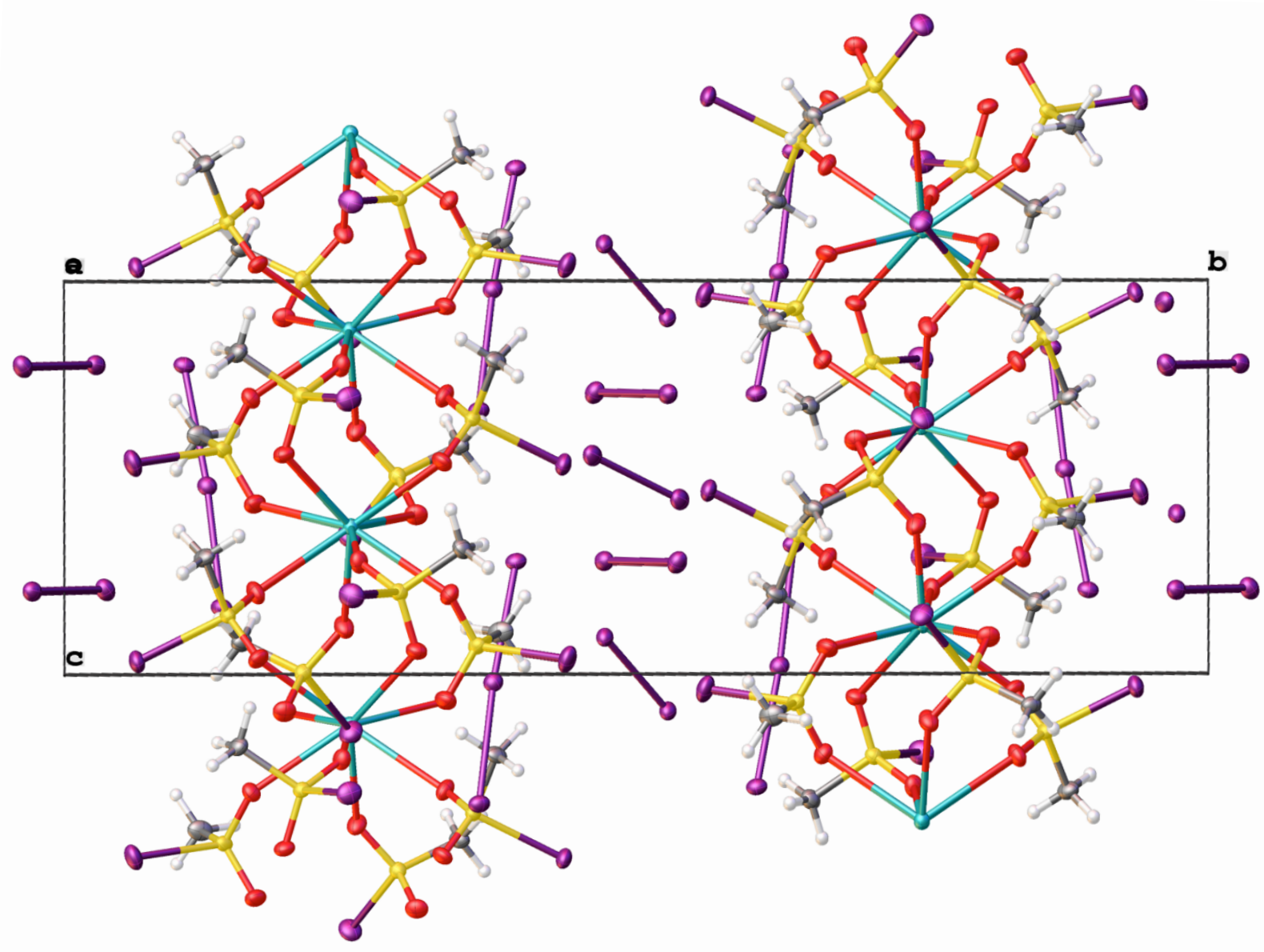

Figure S-4. Molecular packing of $\left(\mathrm{CH}_{3} \mathrm{SO}_{2} \mathrm{I}\right)_{4} \cdot \mathrm{KI}_{3} \cdot 2 \mathrm{I}_{2}$ showing the infinite chain of $\left[\left(\mathrm{CH}_{3} \mathrm{SO}_{2} \mathrm{I}\right)_{4} \cdot \mathrm{K}_{0}\right.$ extending along the $c$-axis. 


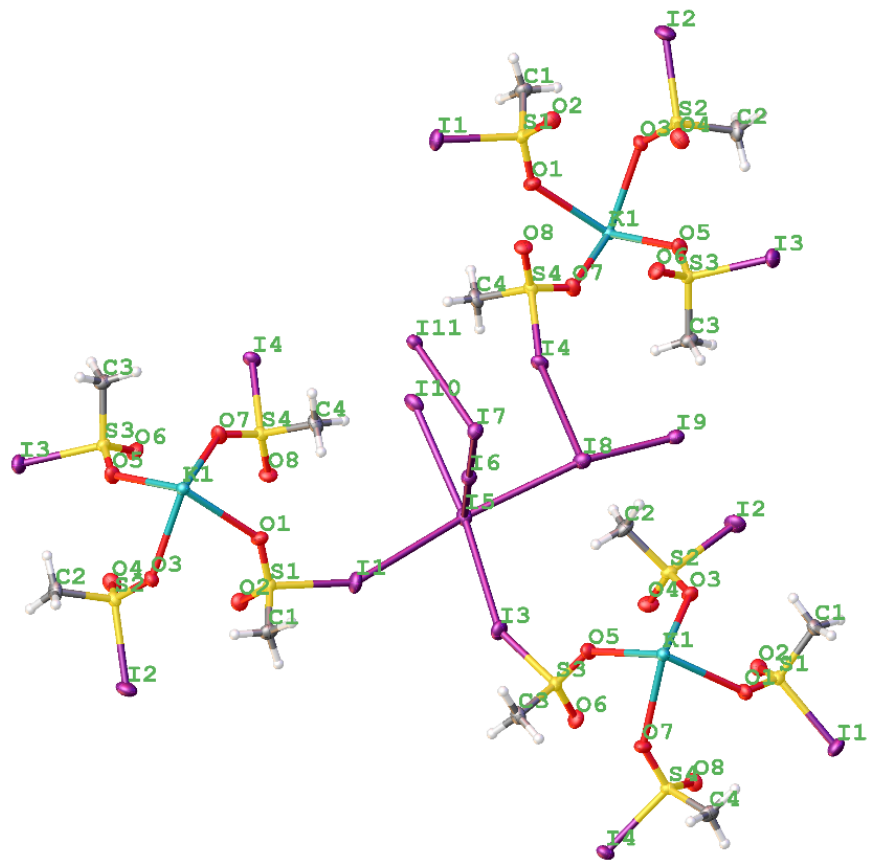

Figure S-4a. Portion of the unit cell of $\left(\mathrm{CH}_{3} \mathrm{SO}_{2} \mathrm{I}\right)_{4} \cdot \mathrm{KI}_{3} \cdot 2 \mathrm{I}_{2}$ showing some of the close I-I contacts between the $\mathrm{CH}_{3} \mathrm{SO}_{2} \mathrm{I}$ and the polyiodide components.

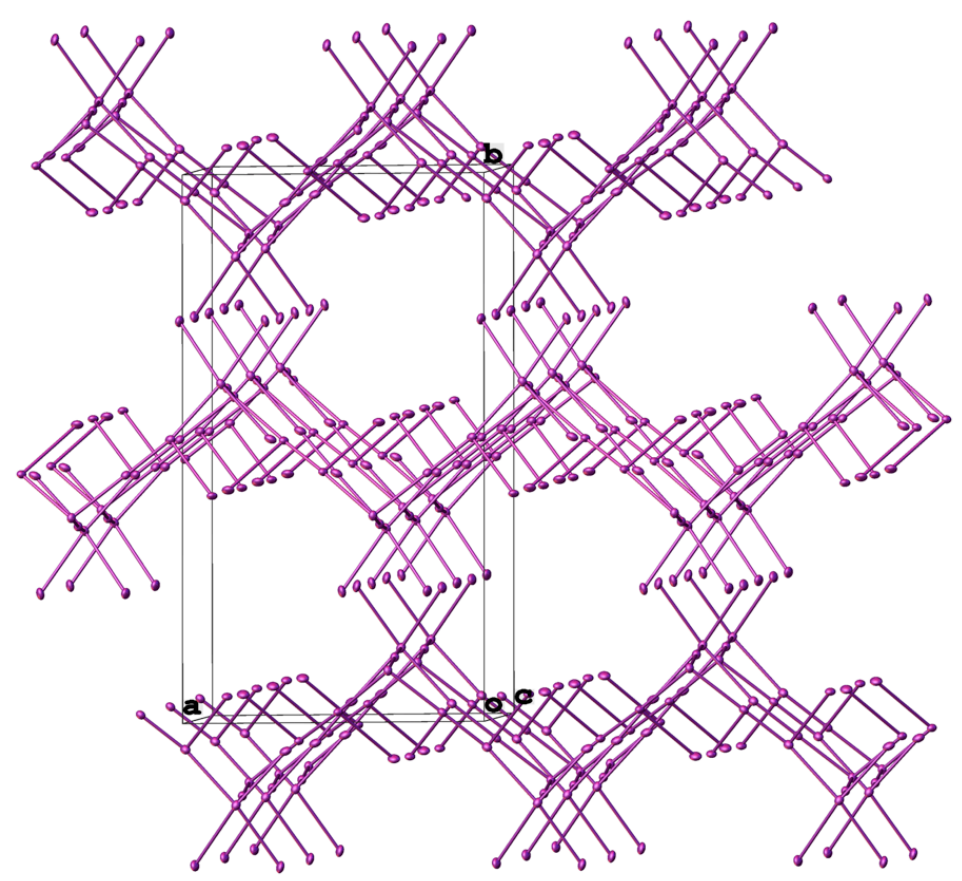

Figure S-5. Molecular packing diagram of $\left(\mathrm{CH}_{3} \mathrm{SO}_{2} \mathrm{I}\right)_{4} \cdot \mathrm{KI}_{3} \cdot 2 \mathrm{I}_{2}$ viewed along the $a b$ plane showing all iodine-iodine contacts less than 4.0 $\AA$. $\left(\mathrm{CH}_{3} \mathrm{SO}_{2}\right)$ and $\mathrm{K}$ removed for clarity. 
Table S10. Sample and crystal data for $\left(\mathrm{CH}_{3} \mathrm{SO}_{2} \mathrm{I}\right)_{4} \cdot \mathrm{KI}_{3} \cdot 2 \mathrm{I}_{2}$.

$\begin{array}{lll}\text { Identification code } & \text { Stanbury072418 } \\ \text { Chemical formula } & \mathrm{C}_{4} \mathrm{H}_{12} \mathrm{I}_{11} \mathrm{KO}_{8} \mathrm{~S}_{4} & \\ \text { Formula weight } & 1751.38 \mathrm{~g} / \mathrm{mol} & \\ \text { Temperature } & 100(2) \mathrm{K} \\ \text { Crystal size } & 0.010 \times 0.011 \times 0.234 \mathrm{~mm} \\ \text { Crystal habit } & \text { dark brown-green needle } \\ \text { Crystal system } & \text { monoclinic } & \\ \text { Space group } & \mathrm{P} 2{ }_{1} / \mathrm{c} & \\ \text { Unit cell dimensions } & \mathrm{a}=14.6211(6) \AA & \alpha=90^{\circ} \\ & \mathrm{b}=26.1593(9) \AA & \beta=108.9673(11)^{\circ} \\ & \mathrm{c}=9.5131(3) \AA & \gamma=90^{\circ} \\ \text { Volume } & 3441.0(2) \AA^{3} & \\ \text { Z } & 4 & \\ \text { Density (calculated) } & 3.381 \mathrm{~g} / \mathrm{cm}^{3} & \\ \text { Absorption coefficient } & 10.290 \mathrm{~mm}^{-1} & \\ \text { F(000) } & 3064\end{array}$

Diffractometer

Bruker D8 VENTURE $\kappa$-geometry diffractometer

Radiation source

Incoatec I $\mu \mathrm{S} 3.0$ microfocus sealed tube (Mo K $\alpha, \lambda=0.71073 \AA$ )

Theta range for data collection 2.26 to $34.35^{\circ}$

Index ranges

$-22<=\mathrm{h}<=23,-41<=\mathrm{k}<=41,-13<=\mathrm{l}<=15$

Reflections collected

198851

Independent reflections

$14349[\mathrm{R}(\mathrm{int})=0.0673]$

Absorption correction

Multi-Scan

Max. and min. transmission

0.9040 and 0.1970

Structure solution technique

direct methods

Structure solution program

Refinement method

SHELXT 2014/5 (Sheldrick, 2014)

Full-matrix least-squares on $\mathrm{F}^{2}$

Refinement program

SHELXL-2017/1 (Sheldrick, 2017)

Function minimized

$\Sigma \mathrm{w}\left(\mathrm{F}_{\mathrm{o}}^{2}-\mathrm{F}_{\mathrm{c}}^{2}\right)^{2}$

Data / restraints / parameters

Goodness-of-fit on $\mathrm{F}^{2}$

$14349 / 0 / 258$

Final $\mathrm{R}$ indices

1.088

12473 data; $\mathrm{I}>2 \sigma(\mathrm{I})$

$\mathrm{R} 1=0.0333, \mathrm{wR} 2=0.0669$

all data

$\mathrm{R} 1=0.0458, \mathrm{wR} 2=0.0728$

Largest diff. peak and hole

2.428 and $-1.947 \mathrm{e}^{-3}$

R.M.S. deviation from mean

$0.275 \mathrm{e}^{-3}$ 
Table S11. Atomic coordinates and equivalent isotropic atomic displacement parameters $\left(\AA^{2}\right)$ for $\left(\mathrm{CH}_{3} \mathrm{SO}_{2} \mathrm{I}\right)_{4} \cdot \mathrm{KI}_{3} \cdot 2 \mathrm{I}_{2}$.

$\mathrm{U}(\mathrm{eq})$ is defined as one third of the trace of the orthogonalized $\mathrm{U}_{\mathrm{ij}}$ tensor.

\begin{tabular}{|c|c|c|c|c|}
\hline & $\mathrm{x} / \mathrm{a}$ & $\mathrm{y} / \mathrm{b}$ & $\mathrm{z} / \mathrm{c}$ & $\mathrm{U}(\mathrm{eq})$ \\
\hline $\mathrm{I} 5$ & $7409.6(3)$ & $8625.0(2)$ & $1695.4(4)$ & $21.72(6)$ \\
\hline I6 & $7506.7(3)$ & $8749.2(2)$ & 4787.4(4) & $20.88(6)$ \\
\hline $\mathrm{I} 7$ & $7583.2(3)$ & $8949.2(2)$ & $7858.1(4)$ & $20.21(6)$ \\
\hline I1 & $5973.2(3)$ & $7488.2(2)$ & $1980.6(5)$ & $27.80(8)$ \\
\hline $\mathrm{I} 2$ & $2538.5(3)$ & $5602.6(2)$ & $369.0(5)$ & $30.87(8)$ \\
\hline $\mathrm{I} 3$ & $-1040.8(3)$ & $7495.6(2)$ & $-1471.7(5)$ & $27.27(8)$ \\
\hline $\mathrm{I} 4$ & 2494.2(3) & $9364.9(2)$ & $323.8(4)$ & $22.71(6)$ \\
\hline K1 & $2473.1(8)$ & $7493.8(4)$ & $3730.7(10)$ & $15.18(14)$ \\
\hline $\mathrm{S} 1$ & 4499.1(9) & $7073.0(5)$ & $2072.0(13)$ & $15.3(2)$ \\
\hline $\mathrm{S} 2$ & $1744.0(9)$ & $6393.3(5)$ & $715.8(13)$ & $16.6(2)$ \\
\hline $\mathrm{S} 3$ & $449.4(9)$ & $7912.2(5)$ & $39.5(13)$ & $15.9(2)$ \\
\hline $\mathrm{S} 4$ & $3261.6(9)$ & $8572.6(5)$ & $1472.3(13)$ & $15.8(2)$ \\
\hline $\mathrm{O} 1$ & $4074(3)$ & $7423.5(15)$ & $2845(5)$ & $21.4(7)$ \\
\hline $\mathrm{O} 2$ & $3949(3)$ & 6921.7(16) & $583(4)$ & $21.9(8)$ \\
\hline $\mathrm{O} 3$ & $2378(3)$ & $6632.8(14)$ & $2022(4)$ & $20.3(7)$ \\
\hline $\mathrm{O} 4$ & $1471(3)$ & $6667.9(16)$ & $-666(4)$ & $25.4(8)$ \\
\hline O5 & 911(3) & $7554.6(16)$ & $1199(5)$ & $24.5(9)$ \\
\hline O6 & $956(3)$ & $8076.3(16)$ & $-941(4)$ & $22.5(8)$ \\
\hline $\mathrm{O} 7$ & $2589(3)$ & $8327.1(14)$ & 2071(4) & $23.3(7)$ \\
\hline O8 & $3572(3)$ & $8303.0(16)$ & $391(4)$ & $22.2(8)$ \\
\hline $\mathrm{C} 1$ & $4892(4)$ & $6521(2)$ & $3171(6)$ & $21.5(10)$ \\
\hline $\mathrm{C} 2$ & $688(4)$ & $6185(3)$ & $1056(7)$ & $25.8(12)$ \\
\hline C3 & $78(4)$ & $8451(2)$ & $816(6)$ & $21.0(10)$ \\
\hline $\mathrm{C} 4$ & $4287(4)$ & $8773(2)$ & $2956(6)$ & 23.1(10) \\
\hline I8 & $9136.5(3)$ & $9640.7(2)$ & $2164.3(4)$ & $23.31(7)$ \\
\hline I9 & $10611.4(3)$ & $10279.6(2)$ & $2120.3(4)$ & $20.36(7)$ \\
\hline I11 & $5744.0(3)$ & $9724.7(2)$ & $5934.3(4)$ & $20.36(7)$ \\
\hline $\mathrm{I} 10$ & $5654.6(3)$ & $9619.2(2)$ & $557.3(5)$ & $26.95(8)$ \\
\hline
\end{tabular}

Table S12. Bond lengths $(\AA)$ for $\left(\mathrm{CH}_{3} \mathrm{SO}_{2} \mathrm{I}\right)_{4} \cdot \mathrm{KI}_{3} \cdot 2 \mathrm{I}_{2}$.
I5 I6 2.9166(5)
$\mathrm{K} 1 \mathrm{~K} 1^{2}$
$4.75665(15)$
I6 I7 2.9337(5)
S1 O1 1.437(4) 


\begin{tabular}{|c|c|c|c|}
\hline I1 S1 & $2.4402(13)$ & $\mathrm{S} 1 \mathrm{O} 2$ & $1.439(4)$ \\
\hline I2 S2 & $2.4465(14)$ & $\mathrm{S} 1 \mathrm{C} 1$ & $1.766(5)$ \\
\hline I3 S3 & $2.4425(13)$ & $\mathrm{S} 2 \mathrm{O} 3$ & $1.431(4)$ \\
\hline I4 S4 & $2.4396(13)$ & $\mathrm{S} 2 \mathrm{O} 4$ & $1.436(4)$ \\
\hline K1 O7 & $2.729(4)$ & $\mathrm{S} 2 \mathrm{C} 2$ & $1.763(6)$ \\
\hline K1 O5 & $2.733(4)$ & S3 O6 & $1.433(4)$ \\
\hline K1O1 & $2.739(4)$ & S3 O5 & $1.436(4)$ \\
\hline K1O3 & $2.755(4)$ & S3 C3 & $1.757(5)$ \\
\hline $\mathrm{K} 1 \mathrm{O} 2^{1}$ & $2.761(4)$ & S4 O8 & $1.437(4)$ \\
\hline $\mathrm{K} 1 \mathrm{O} 6^{1}$ & $2.773(4)$ & S4 O7 & $1.438(4)$ \\
\hline $\mathrm{K} 1 \mathrm{O} 8^{1}$ & $2.787(4)$ & $\mathrm{S} 4 \mathrm{C} 4$ & $1.772(5)$ \\
\hline $\mathrm{K} 1 \mathrm{O} 4^{1}$ & $2.799(4)$ & I8 I9 & $2.7393(5)$ \\
\hline $\mathrm{K} 1 \mathrm{~S} 3^{1}$ & $3.7187(16)$ & $\mathrm{I} 11 \mathrm{I} 11^{3}$ & $2.7301(7)$ \\
\hline $\mathrm{K} 1 \mathrm{~S} 4{ }^{1}$ & $3.7361(16)$ & $\mathrm{I} 10 \mathrm{I} 10^{4}$ & $2.7273(9)$ \\
\hline $\mathrm{K} 1 \mathrm{~S} 1^{1}$ & $3.7472(16)$ & & \\
\hline
\end{tabular}

Table S13. Bond angles $\left({ }^{\circ}\right)$ for $\left(\mathrm{CH}_{3} \mathrm{SO}_{2} \mathrm{I}\right)_{4} \cdot \mathrm{KI}_{3} \cdot 2 \mathrm{I}_{2}$.

\begin{tabular}{|c|c|c|c|}
\hline I5 $\quad$ I6 $\mathrm{I} 7$ & $176.089(16)$ & $\mathrm{O} 7 \mathrm{~K} 1 \mathrm{~K} 1^{2}$ & $52.83(9)$ \\
\hline O7 K1O5 & $68.58(12)$ & $\mathrm{O} 5 \mathrm{~K} 1 \mathrm{~K} 1^{2}$ & $52.39(10)$ \\
\hline $\mathrm{O} 7 \mathrm{~K} 1 \mathrm{O} 1$ & $69.86(13)$ & $\mathrm{O} 1 \mathrm{~K} 1 \mathrm{~K} 1^{2}$ & $54.21(9)$ \\
\hline O5 K1O1 & $106.60(12)$ & $\mathrm{O} 3 \mathrm{~K} 1 \mathrm{~K} 1^{2}$ & $55.38(8)$ \\
\hline O7 K1 O3 & $108.20(11)$ & $\mathrm{O} 2^{1} \mathrm{~K} 1 \mathrm{~K} 1^{2}$ & $112.35(9)$ \\
\hline $\mathrm{O} 5 \mathrm{~K} 1 \mathrm{O} 3$ & $70.12(12)$ & $\mathrm{O} 6^{1} \mathrm{~K} 1 \mathrm{~K} 1^{2}$ & $112.09(9)$ \\
\hline $\mathrm{O} 1 \mathrm{~K} 1 \mathrm{O} 3$ & $69.39(12)$ & $\mathrm{O} 8^{1} \mathrm{~K} 1 \mathrm{~K} 1^{2}$ & 112.61(9) \\
\hline $\mathrm{O} 7 \mathrm{~K} 1 \mathrm{O} 2^{1}$ & $75.18(12)$ & $\mathrm{O} 4{ }^{1} \mathrm{~K} 1 \mathrm{~K} 1^{2}$ & 111.71(9) \\
\hline $\mathrm{O} 5 \mathrm{~K} 1 \mathrm{O} 2^{1}$ & $141.81(13)$ & $\mathrm{S} 3^{1} \mathrm{~K} 1 \mathrm{~K} 1^{2}$ & $126.54(3)$ \\
\hline $\mathrm{O} 1 \mathrm{~K} 1 \mathrm{O} 2^{1}$ & $70.43(13)$ & $\mathrm{S} 4^{1} \mathrm{~K} 1 \mathrm{~K} 1^{2}$ & $127.07(4)$ \\
\hline $\mathrm{O} 3 \mathrm{~K} 1 \mathrm{O} 2^{1}$ & $135.11(12)$ & $\mathrm{S} 1^{1} \mathrm{~K} 1 \mathrm{~K} 1^{2}$ & $126.10(3)$ \\
\hline O7 K1 O6 ${ }^{1}$ & $134.19(13)$ & $\mathrm{O} 1 \mathrm{~S} 1 \mathrm{O} 2$ & $118.9(2)$ \\
\hline $\mathrm{O} 5 \mathrm{~K} 1 \mathrm{O} 6^{1}$ & $70.16(13)$ & O1 S1 C1 & $109.3(3)$ \\
\hline $\mathrm{O} 1 \mathrm{~K} 1 \mathrm{O} 6^{1}$ & $142.47(13)$ & $\mathrm{O} 2 \mathrm{~S} 1 \mathrm{C} 1$ & $109.2(3)$ \\
\hline $\mathrm{O} 3 \mathrm{~K}^{10} 6^{1}$ & $74.80(12)$ & O1 S1 I1 & $105.56(17)$ \\
\hline $\mathrm{O} 2{ }^{1} \mathrm{~K} 1 \mathrm{O} 6{ }^{1}$ & $135.53(11)$ & $\mathrm{O} 2 \mathrm{~S} 1 \mathrm{I} 1$ & $107.77(18)$ \\
\hline $\mathrm{O} 7 \mathrm{~K} 1 \mathrm{O} 8^{1}$ & $142.40(13)$ & C1 S1 I1 & $105.30(19)$ \\
\hline $\mathrm{O} 5 \mathrm{~K} 1 \mathrm{O} 8^{1}$ & $134.87(13)$ & $\mathrm{O} 1 \mathrm{~S} 1 \mathrm{~K} 1^{2}$ & $83.51(18)$ \\
\hline $\mathrm{O} 1 \mathrm{~K} 1 \mathrm{O} 8^{1}$ & $74.42(12)$ & $\mathrm{O} 2 \mathrm{~S} 1 \mathrm{~K} 1^{2}$ & $38.09(17)$ \\
\hline $\mathrm{O} 3 \mathrm{~K} 1 \mathrm{O} 8^{1}$ & $68.42(12)$ & C1 S1 K $1^{2}$ & $139.90(19)$ \\
\hline $\mathrm{O} 2^{1} \mathrm{~K} 1 \mathrm{O} 8^{1}$ & $82.29(12)$ & $\mathrm{I} 1 \mathrm{~S} 1 \mathrm{~K} 1^{2}$ & $107.40(4)$ \\
\hline
\end{tabular}




\begin{tabular}{|c|c|c|c|}
\hline $\mathrm{O} 6^{1} \mathrm{~K} 1 \mathrm{O} 8^{1}$ & $82.46(12)$ & $\mathrm{O} 3 \mathrm{~S} 2 \mathrm{O} 4$ & $119.0(2)$ \\
\hline $\mathrm{O} 7 \mathrm{~K} 1 \mathrm{O} 4^{1}$ & $68.16(13)$ & $\mathrm{O} 3 \mathrm{~S} 2 \mathrm{C} 2$ & $109.6(3)$ \\
\hline $\mathrm{O} 5 \mathrm{~K} 1 \mathrm{O} 4^{1}$ & $77.99(13)$ & $\mathrm{O} 4 \mathrm{~S} 2 \mathrm{C} 2$ & $108.9(3)$ \\
\hline $\mathrm{O} 1 \mathrm{~K} 1 \mathrm{O} 4^{1}$ & $132.25(13)$ & $\mathrm{O} 3 \mathrm{~S} 2 \mathrm{I} 2$ & $106.58(17)$ \\
\hline $\mathrm{O} 3 \mathrm{~K} \mathrm{O} 4^{1}$ & $146.34(13)$ & $\mathrm{O} 4 \mathrm{~S} 2 \mathrm{I} 2$ & $107.60(19)$ \\
\hline $\mathrm{O} 2^{1} \mathrm{~K} 1 \mathrm{O} 4^{1}$ & $77.74(13)$ & $\mathrm{C} 2 \mathrm{~S} 2 \mathrm{I} 2$ & $104.2(2)$ \\
\hline $\mathrm{O} 6{ }^{1} \mathrm{~K} 1 \mathrm{O} 4^{1}$ & $84.67(13)$ & O6 S3 O5 & $118.8(3)$ \\
\hline $\mathrm{O} 8{ }^{1} \mathrm{~K} 1 \mathrm{O} 4^{1}$ & $135.52(11)$ & O6 S3 C3 & 109.2(3) \\
\hline O7 K1 S3 ${ }^{1}$ & $128.88(10)$ & O5 S3 C3 & $109.4(3)$ \\
\hline $\mathrm{O} 5 \mathrm{~K} 1 \mathrm{~S} 3^{1}$ & $77.51(10)$ & O6 S3 I3 & $107.55(18)$ \\
\hline $\mathrm{O} 1 \mathrm{~K} 1 \mathrm{~S} 3^{1}$ & $159.42(9)$ & O5 S3 I3 & $105.54(17)$ \\
\hline $\mathrm{O} 3 \mathrm{~K} 1 \mathrm{~S} 3^{1}$ & $94.07(9)$ & C3 S3 I3 & $105.46(19)$ \\
\hline $\mathrm{O} 2{ }^{1} \mathrm{~K} 1 \mathrm{~S} 3^{1}$ & $119.09(9)$ & O6 S3 K1 ${ }^{2}$ & $39.77(17)$ \\
\hline $\mathrm{O} 6{ }^{1} \mathrm{~K} 1 \mathrm{~S} 3^{1}$ & $19.30(9)$ & O5 S3 K1 $1^{2}$ & $81.04(19)$ \\
\hline $\mathrm{O} 8^{1} \mathrm{~K} 1 \mathrm{~S} 3^{1}$ & $88.36(9)$ & C $3 \mathrm{~S} 3 \mathrm{~K} 1^{2}$ & $139.3(2)$ \\
\hline $\mathrm{O} 4{ }^{1} \mathrm{~K} 1 \mathrm{~S} 3^{1}$ & $68.19(10)$ & I3 S3 K1 ${ }^{2}$ & $109.28(4)$ \\
\hline $\mathrm{O} 7 \mathrm{~K} 1 \mathrm{~S} 4^{1}$ & $159.58(10)$ & O8 S4 O7 & $118.7(2)$ \\
\hline O5 K1 S4 ${ }^{1}$ & $129.69(10)$ & $\mathrm{O} 8 \mathrm{~S} 4 \mathrm{C} 4$ & $109.5(3)$ \\
\hline $\mathrm{O} 1 \mathrm{~K} 1 \mathrm{~S} 4^{1}$ & $93.66(9)$ & $\mathrm{O} 7 \mathrm{~S} 4 \mathrm{C} 4$ & 109.1(3) \\
\hline $\mathrm{O} 3 \mathrm{~K} 1 \mathrm{~S} 4^{1}$ & $75.30(8)$ & O8 S4 I4 & $107.62(18)$ \\
\hline $\mathrm{O} 2{ }^{1} \mathrm{~K} 1 \mathrm{~S} 4^{1}$ & $88.19(9)$ & $\mathrm{O} 7 \mathrm{~S} 4 \mathrm{I} 4$ & $106.42(17)$ \\
\hline $\mathrm{O} 6{ }^{1} \mathrm{~K} 1 \mathrm{~S} 4^{1}$ & $66.21(9)$ & $\mathrm{C} 4 \mathrm{~S} 4 \mathrm{I} 4$ & 104.6(2) \\
\hline $\mathrm{O} 8^{1} \mathrm{~K} 1 \mathrm{~S} 4^{1}$ & $19.25(9)$ & O8 S4 K1 $1^{2}$ & $39.76(17)$ \\
\hline $\mathrm{O} 4{ }^{1} \mathrm{~K} 1 \mathrm{~S} 4{ }^{1}$ & $120.35(9)$ & O7 S4 K1 ${ }^{2}$ & $81.22(17)$ \\
\hline $\mathrm{S} 3^{1} \mathrm{~K} 1 \mathrm{~S} 4^{1}$ & 69.61(3) & $\mathrm{C} 4 \mathrm{~S} 4 \mathrm{~K} 1^{2}$ & $140.0(2)$ \\
\hline O7 K1 S1 ${ }^{1}$ & 93.93(9) & I4 S4 K1 ${ }^{2}$ & $109.21(4)$ \\
\hline O5 K1 S1 ${ }^{1}$ & $159.05(10)$ & S1 O1 K1 & $139.1(2)$ \\
\hline $\mathrm{O} 1 \mathrm{~K} 1 \mathrm{~S} 1^{1}$ & $76.25(10)$ & $\mathrm{S} 1 \mathrm{O} 2 \mathrm{~K}^{2}$ & $123.2(2)$ \\
\hline $\mathrm{O} 3 \mathrm{~K} 1 \mathrm{~S} 1^{1}$ & $128.48(9)$ & S2 O3 K1 & $139.9(2)$ \\
\hline $\mathrm{O} 2{ }^{1} \mathrm{~K} 1 \mathrm{~S} 1^{1}$ & $18.76(9)$ & $\mathrm{S} 2 \mathrm{O} 4 \mathrm{~K} 1^{2}$ & $124.8(2)$ \\
\hline $\mathrm{O} 6{ }^{1} \mathrm{~K} 1 \mathrm{~S} 1^{1}$ & $120.52(9)$ & S3 O5K1 & $139.8(2)$ \\
\hline $\mathrm{O} 8{ }^{1} \mathrm{~K} 1 \mathrm{~S} 1^{1}$ & 66.07(9) & $\mathrm{S} 3 \mathrm{O} 6 \mathrm{~K} 1^{2}$ & $120.9(2)$ \\
\hline $\mathrm{O} 4{ }^{1} \mathrm{~K} 1 \mathrm{~S} 1{ }^{1}$ & $84.91(9)$ & S4 O7K1 & $139.9(2)$ \\
\hline $\mathrm{S} 3^{1} \mathrm{~K} 1 \mathrm{~S} 1^{1}$ & $107.34(3)$ & $\mathrm{S} 4 \mathrm{O} 8 \mathrm{~K} 1^{2}$ & $121.0(2)$ \\
\hline $\mathrm{S} 4{ }^{1} \mathrm{~K} 1 \mathrm{~S} 1^{1}$ & $69.77(3)$ & & \\
\hline
\end{tabular}

Table S14. Torsion angles $\left({ }^{\circ}\right)$ for $\left(\mathrm{CH}_{3} \mathrm{SO}_{2} \mathrm{I}\right)_{4} \cdot \mathrm{KI}_{3} \cdot 2 \mathrm{I}_{2}$.
A B C D Angle $/^{\circ}$
A B C D Angle $/^{\circ}$
O2 S1 O1 K1 58.0(4)
C3 S3 O5 K1 -61.1(5)
C1 S1O1 K1 -68.1(4)
I3 $\mathrm{S} 3 \mathrm{O} 5 \mathrm{~K} 1 \quad-174.2(3)$ 


\begin{tabular}{|c|c|c|c|}
\hline I1 S1O1K1 & 179.1(3) & $\mathrm{K} 1{ }^{1} \mathrm{~S} 3 \mathrm{O} 5 \mathrm{~K} 1$ & $78.2(4)$ \\
\hline $\mathrm{K} 1{ }^{1} \mathrm{~S} 1 \mathrm{O} 1 \mathrm{~K} 1$ & $72.8(3)$ & $\mathrm{O} 5 \mathrm{~S} 3 \mathrm{O} 6 \mathrm{~K} 1^{1}$ & $20.3(4)$ \\
\hline $\mathrm{O} 1 \mathrm{~S} 1 \mathrm{O} 2 \mathrm{~K} 1^{1}$ & $24.2(4)$ & C3 S3O6K $1^{1}$ & $146.7(2)$ \\
\hline $\mathrm{C} 1 \mathrm{~S} 1 \mathrm{O} 2 \mathrm{~K} 1^{1}$ & $150.4(3)$ & I3 S3 O6 K $1^{1}$ & $-99.3(2)$ \\
\hline I1 $\mathrm{S} 1 \mathrm{O} 2 \mathrm{~K} 1^{1}$ & $-95.7(2)$ & O8 S4O7K1 & $63.6(4)$ \\
\hline O4 S2O3 K1 & $58.6(4)$ & C4 S4O7K1 & $-62.7(4)$ \\
\hline C2 S2O3 K1 & $-67.5(4)$ & I4 S4O7K1 & $-175.0(3)$ \\
\hline I2 S2O3 K1 & $-179.7(3)$ & $\mathrm{K} 1{ }^{1} \mathrm{~S} 4 \mathrm{O} 7 \mathrm{~K} 1$ & $77.4(3)$ \\
\hline $\mathrm{O} 3 \mathrm{~S}_{2} \mathrm{O} 4 \mathrm{~K} 1^{1}$ & $19.5(4)$ & $\mathrm{O} 7 \mathrm{~S} 4 \mathrm{O} 8 \mathrm{~K} 1^{1}$ & $21.7(3)$ \\
\hline $\mathrm{C} 2 \mathrm{~S} 2 \mathrm{O} 4 \mathrm{~K} 1^{1}$ & $145.9(3)$ & $\mathrm{C} 4 \mathrm{~S} 4 \mathrm{O} 8 \mathrm{~K} 1^{1}$ & $147.7(3)$ \\
\hline $\mathrm{I} 2 \mathrm{~S} 2 \mathrm{O} 4 \mathrm{~K} 1^{1}$ & $-101.7(2)$ & I4 $\mathrm{S} 4 \mathrm{O} 8 \mathrm{~K} 1^{1}$ & $-99.1(2)$ \\
\hline O6 S3O5 K1 & $65.2(5)$ & & \\
\hline
\end{tabular}

Table S15. Anisotropic atomic displacement parameters $\left(\AA^{2}\right)$ for $\left(\mathrm{CH}_{3} \mathrm{SO}_{2} \mathrm{I}\right)_{4} \cdot \mathrm{KI}_{3} \cdot 2 \mathrm{I}_{2}$.

The anisotropic atomic displacement factor exponent takes the form:

\begin{tabular}{|c|c|c|c|c|c|c|}
\hline Atom & $\mathrm{U}_{11}$ & $\mathrm{U}_{22}$ & $\mathrm{U}_{33}$ & $\mathrm{U}_{12}$ & $\mathrm{U}_{13}$ & $\mathrm{U}_{23}$ \\
\hline I5 & $22.04(15)$ & $26.03(15)$ & $17.25(14)$ & $-3.40(13)$ & $6.61(13)$ & $1.46(11)$ \\
\hline I6 & $19.31(13)$ & $21.96(13)$ & $20.74(14)$ & $0.00(12)$ & $5.64(13)$ & $1.24(11)$ \\
\hline I7 & $18.80(14)$ & $25.02(14)$ & $15.95(13)$ & $1.29(12)$ & $4.45(12)$ & $1.63(11)$ \\
\hline I1 & $17.39(15)$ & $36.3(2)$ & $30.62(19)$ & $-4.78(13)$ & $9.08(16)$ & $2.58(15)$ \\
\hline $\mathrm{I} 2$ & $39.9(2)$ & $19.07(15)$ & $36.2(2)$ & $-1.39(16)$ & $15.9(2)$ & $-8.35(14)$ \\
\hline I3 & $17.86(14)$ & $30.9(2)$ & $27.88(18)$ & $-2.82(13)$ & $0.40(15)$ & $-4.33(14)$ \\
\hline I4 & $27.09(15)$ & $14.64(12)$ & $23.84(16)$ & $0.62(13)$ & $4.75(16)$ & $3.44(11)$ \\
\hline K1 & $15.7(4)$ & $14.5(3)$ & $15.5(4)$ & $0.2(3)$ & $5.3(4)$ & $0.0(3)$ \\
\hline S1 & $14.3(5)$ & $16.9(5)$ & $14.6(5)$ & $1.3(4)$ & 4.6(4) & $1.4(4)$ \\
\hline $\mathrm{S} 2$ & $19.8(5)$ & $16.9(5)$ & $14.0(5)$ & $-4.5(4)$ & $6.8(4)$ & $-1.2(4)$ \\
\hline S3 & $14.4(5)$ & $16.7(5)$ & $15.9(5)$ & $1.6(4)$ & $4.0(4)$ & $-0.5(4)$ \\
\hline S4 & $19.0(5)$ & $14.2(5)$ & $14.1(5)$ & $-2.1(4)$ & 5.1(4) & $0.4(4)$ \\
\hline $\mathrm{O} 1$ & 23.1(17) & 18.1(17) & $26(2)$ & $4.2(14)$ & $11.6(16)$ & $1.0(15)$ \\
\hline $\mathrm{O} 2$ & $23.0(18)$ & $25(2)$ & $14.5(16)$ & $1.0(15)$ & $2.5(14)$ & $2.6(14)$ \\
\hline $\mathrm{O} 3$ & $19.7(17)$ & $19.3(16)$ & $21.0(17)$ & $-3.9(14)$ & $5.5(14)$ & $-4.1(13)$ \\
\hline $\mathrm{O} 4$ & $33(2)$ & $26(2)$ & $17.0(17)$ & $-4.8(17)$ & $8.0(16)$ & $2.7(15)$ \\
\hline O5 & $20.0(18)$ & $22.0(19)$ & $25(2)$ & $-0.3(15)$ & $-1.9(16)$ & $4.0(15)$ \\
\hline O6 & $20.8(18)$ & $30(2)$ & $20.9(18)$ & $1.8(15)$ & $11.8(15)$ & $-0.6(15)$ \\
\hline $\mathrm{O} 7$ & $22.6(18)$ & $19.1(16)$ & $25.6(19)$ & $-2.8(15)$ & $4.5(16)$ & $7.2(14)$ \\
\hline $\mathrm{O} 8$ & $27(2)$ & $22.4(19)$ & $17.2(17)$ & $2.1(15)$ & $7.9(15)$ & $-5.8(14)$ \\
\hline $\mathrm{C} 1$ & $23(2)$ & $21(2)$ & $20(2)$ & $4.8(19)$ & $5.4(19)$ & $3.2(18)$ \\
\hline $\mathrm{C} 2$ & $21(2)$ & $36(3)$ & $24(3)$ & $-9(2)$ & $11(2)$ & $-2(2)$ \\
\hline
\end{tabular}




$\begin{array}{lrrrrrr}\text { C3 } & 22(2) & 24(3) & 17(2) & 5.0(19) & 6.1(18) & -1.6(19) \\ \text { C4 } & 24(2) & 26(3) & 17(2) & -8(2) & 3.7(19) & -3.9(19) \\ \text { I8 } & 26.31(16) & 21.58(16) & 24.27(17) & 2.65(13) & 11.30(14) & 3.91(12) \\ \text { I9 } & 22.49(15) & 19.98(15) & 18.87(15) & 3.99(12) & 7.09(12) & 0.57(12) \\ \text { I11 } & 21.98(15) & 16.47(14) & 19.97(15) & -1.17(11) & 3.17(12) & 0.13(11) \\ \text { I10 } & 37.2(2) & 20.47(16) & 25.26(17) & -8.01(14) & 13.08(15) & -0.73(13)\end{array}$

Table S16. Hydrogen bond distances $(\AA)$ and angles $\left({ }^{\circ}\right)$ for $\left(\mathrm{CH}_{3} \mathrm{SO}_{2} \mathrm{I}\right)_{4} \cdot \mathrm{KI}_{3} \cdot 2 \mathrm{I}_{2}$.

\begin{tabular}{|c|c|c|c|c|}
\hline D $\mathrm{H} \quad \mathrm{A}$ & $\mathrm{d}(\mathrm{D}-\mathrm{H}) / \AA$ & $\mathrm{d}(\mathrm{H}-\mathrm{A}) / \AA$ & $\mathrm{d}(\mathrm{D}-\mathrm{A}) / \AA$ & D-H-A/ ${ }^{\circ}$ \\
\hline $\mathrm{C} 1 \mathrm{H} 1 \mathrm{~B} \mathrm{I} 5^{1}$ & 0.98 & 3.28 & $4.112(5)$ & 144.1 \\
\hline $\mathrm{C} 2 \mathrm{H} 2 \mathrm{~A} \mathrm{I} 8^{2}$ & 0.98 & 3.27 & $4.249(6)$ & 173.1 \\
\hline $\mathrm{C} 2 \mathrm{H} 2 \mathrm{C} \mathrm{I} 9^{3}$ & 0.98 & 3.16 & $3.791(6)$ & 123.5 \\
\hline $\mathrm{C} 3 \mathrm{H} 3 \mathrm{C} \mathrm{I} 7^{4}$ & 0.98 & 3.16 & $4.031(5)$ & 148.4 \\
\hline C4 H4AI10 & 0.98 & 3.18 & $4.132(6)$ & 165.2 \\
\hline C4 H4C I1 1 & 0.98 & 3.30 & $3.852(5)$ & 117.2 \\
\hline
\end{tabular}

Table S17. Hydrogen atomic coordinates and isotropic atomic displacement parameters $\left(\AA^{2}\right)$ for $\left(\mathrm{CH}_{3} \mathrm{SO}_{2} \mathrm{I}\right)_{4} \cdot \mathrm{KI}_{3} \cdot 2 \mathrm{I}_{2}$.

\begin{tabular}{lrrrl} 
Atom & \multicolumn{1}{l}{$x$} & \multicolumn{1}{l}{$y$} & \multicolumn{1}{l}{$z$} & $\mathrm{U}(\mathrm{eq})$ \\
H1A & 4331.25 & 6339.69 & 3275.73 & 32 \\
H1B & 5325.49 & 6620.21 & 4155.22 & 32 \\
H1C & 5238.31 & 6295.86 & 2690.96 & 32 \\
H2A & 295.51 & 5980.45 & 211.79 & 39 \\
H2B & 315.42 & 6481.95 & 1184.72 & 39 \\
H2C & 867.49 & 5975.87 & 1959.92 & 39 \\
H3A & 647.23 & 8643.53 & 1408.28 & 32 \\
H3B & -293.53 & 8340.43 & 1453.58 & 32 \\
H3C & -328.01 & 8668.8 & 17.89 & 32 \\
H4A & 4725.88 & 8963.38 & 2558.43 & 35 \\
H4B & 4623.06 & 8472.85 & 3501.12 & 35 \\
H4C & 4080.17 & 8993.23 & 3629.55 & 35
\end{tabular}




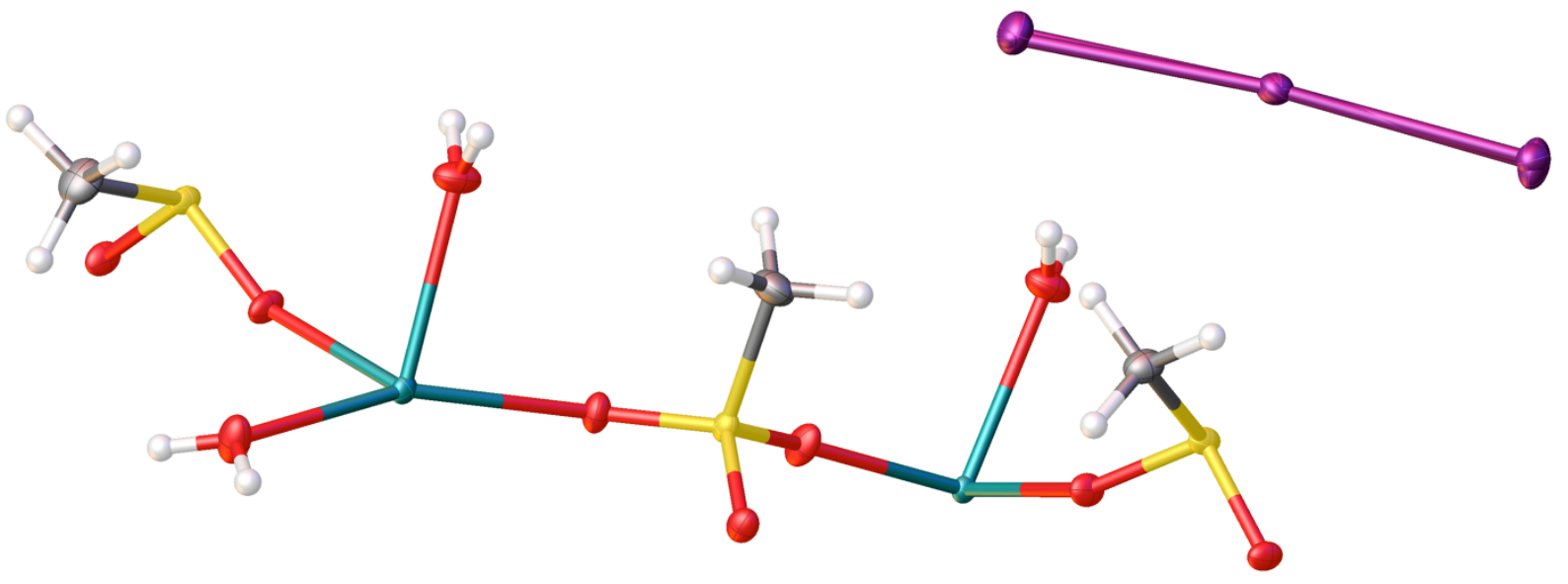

Figure S-6. Asymmetric unit $\mathrm{Ca}_{2}\left(\mathrm{CH}_{3} \mathrm{SO}_{2}\right)_{2}\left(\mathrm{CH}_{3} \mathrm{SO}_{3}\right) \cdot\left(\mathrm{I}_{3}\right) \cdot\left(\mathrm{H}_{2} \mathrm{O}\right)_{3} \cdot$

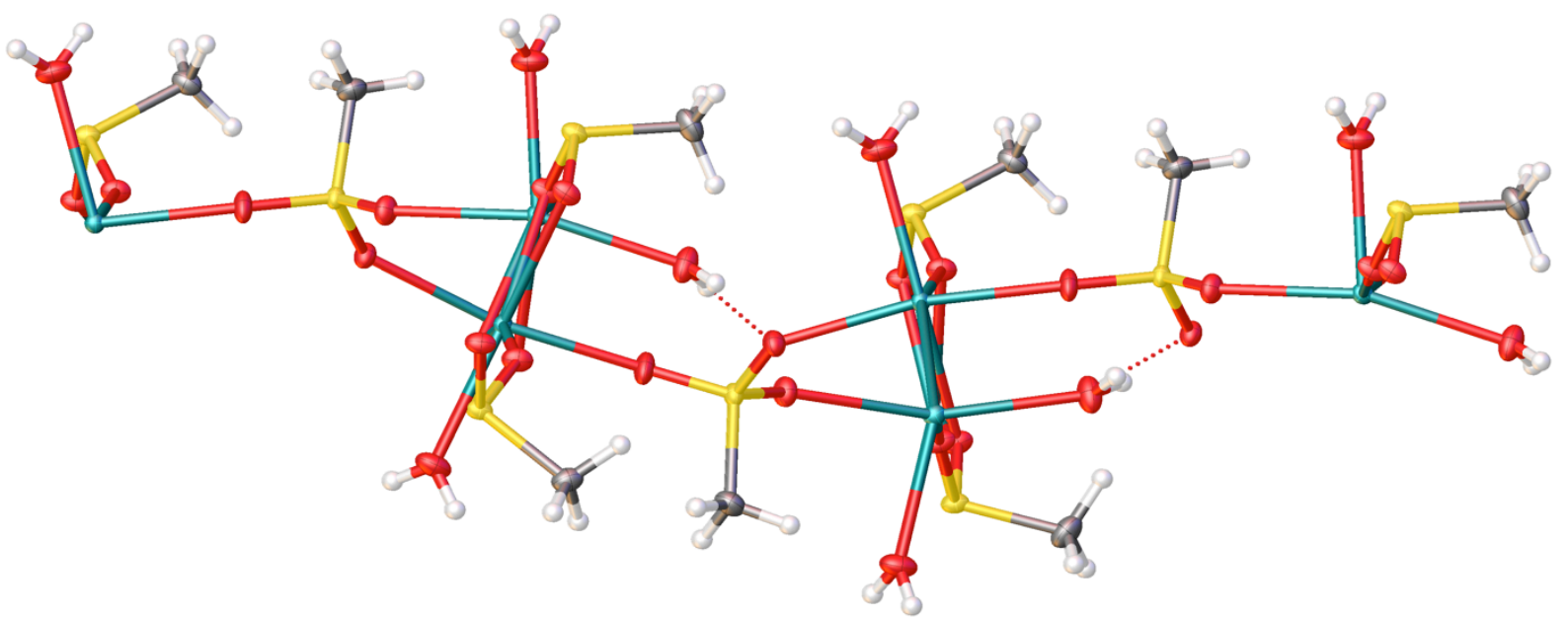

Figure S-7. Portion of $\mathrm{Ca}_{2}\left(\mathrm{CH}_{3} \mathrm{SO}_{2}\right)_{2}\left(\mathrm{CH}_{3} \mathrm{SO}_{3}\right)\left(\mathrm{I}_{3}\right) \cdot\left(\mathrm{H}_{2} \mathrm{O}\right)_{3}$ showing hydrogen bonding. 


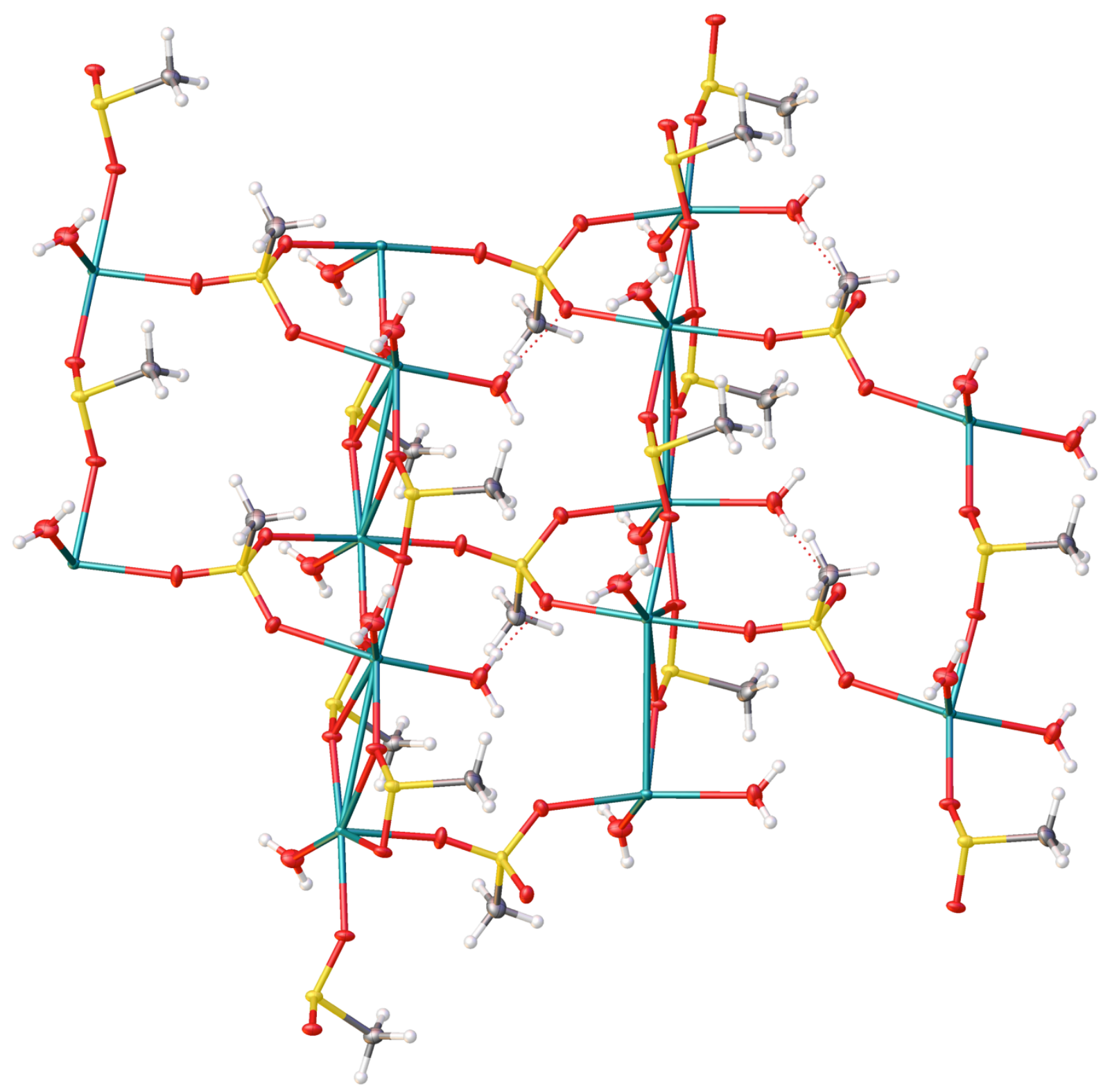

Figure S-8. Grown asymmetric unit of $\mathrm{Ca}_{2}\left(\mathrm{CH}_{3} \mathrm{SO}_{2}\right)_{2}\left(\mathrm{CH}_{3} \mathrm{SO}_{3}\right)\left(\mathrm{I}_{3}\right) \cdot\left(\mathrm{H}_{2} \mathrm{O}\right)_{3}$ showing network connectivity and hydrogen bonding. The $\mathrm{I}_{3}{ }^{-}$is eliminated for clarity. 


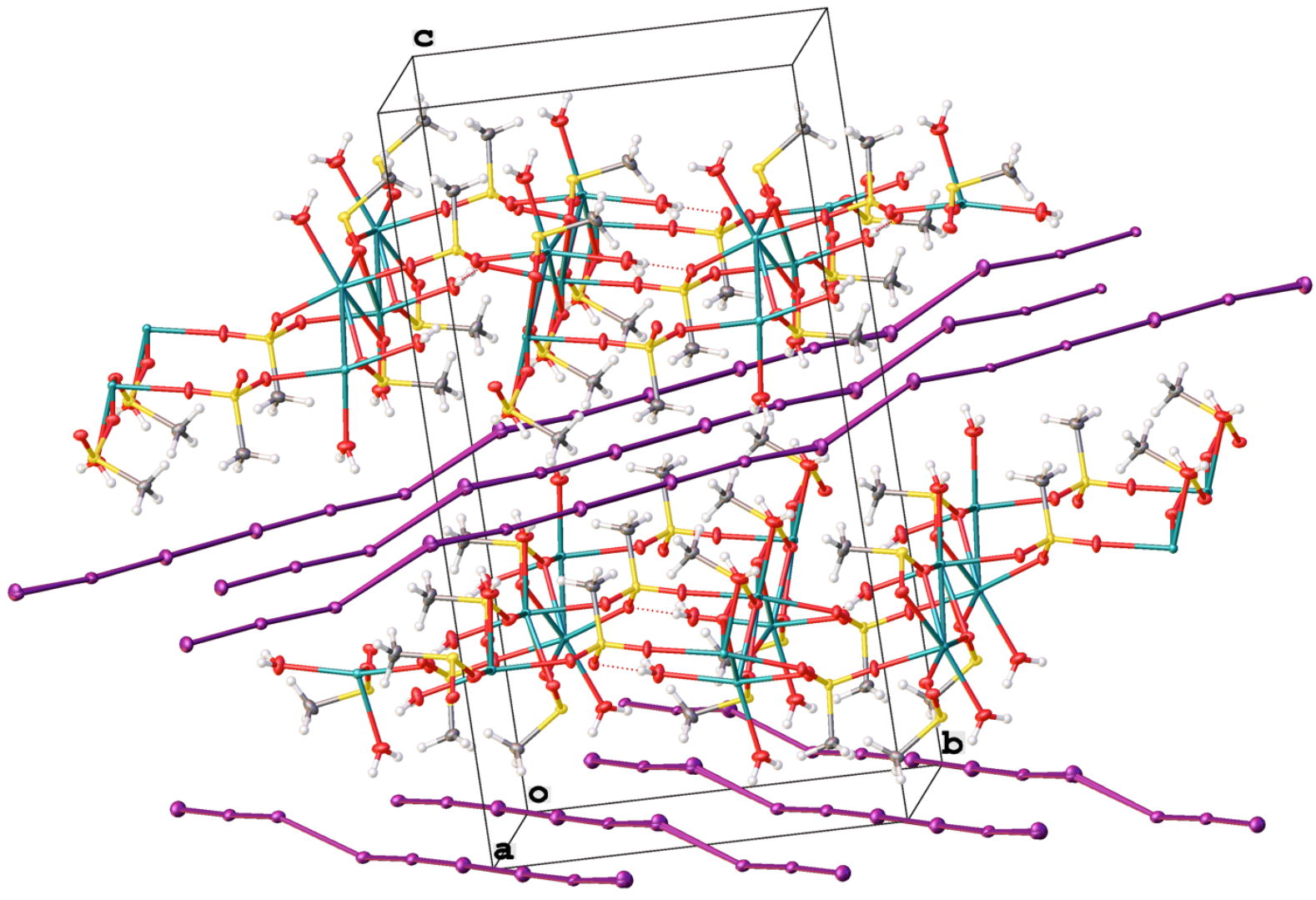

Figure S-9. Molecular packing of $\mathrm{Ca}_{2}\left(\mathrm{CH}_{3} \mathrm{SO}_{2}\right)_{2}\left(\mathrm{CH}_{3} \mathrm{SO}_{3}\right)\left(\mathrm{I}_{3}\right) \cdot\left(\mathrm{H}_{2} \mathrm{O}\right)_{3}$ showing the twodimensional polyiodide sheeting. 
Table S18. Crystal data and structure refinement for $\mathrm{Ca}_{2}\left(\mathrm{CH}_{3} \mathrm{SO}_{2}\right)_{2}\left(\mathrm{CH}_{3} \mathrm{SO}_{3}\right)\left(\mathrm{I}_{3}\right) \cdot\left(\mathrm{H}_{2} \mathrm{O}\right)_{3}$.

Identification code

Chemical formula

Formula weight

Temperature

Crystal size

Crystal habit

Crystal system

Space group

Unit cell dimensions

Volume

Z

Density (calculated)

Absorption coefficient

$\mathrm{F}(000)$

Diffractometer

Radiation source
Stanbury010418

$\mathrm{C}_{3} \mathrm{H}_{15} \mathrm{Ca}_{2} \mathrm{I}_{3} \mathrm{O}_{10} \mathrm{~S}_{3}$

$768.19 \mathrm{~g} / \mathrm{mol}$

100(2) K

$0.040 \times 0.063 \times 0.536 \mathrm{~mm}$

clear pale blue-brown rod

monoclinic

$\mathrm{P} 21 / \mathrm{n}$

$$
\begin{array}{ll}
\mathrm{a}=6.8708(3) \AA & \alpha=90^{\circ} \\
\mathrm{b}=12.7278(6) \AA & \beta=92.486(2)^{\circ} \\
\mathrm{c}=23.3016(11) \AA & \gamma=90^{\circ}
\end{array}
$$

2035.81(16) $\AA^{3}$

4

$2.506 \mathrm{~g} / \mathrm{cm}^{3}$

$5.449 \mathrm{~mm}^{-1}$

1440

Bruker D8 VENTURE $\kappa$-geometry diffractometer

Incoatec $\mathrm{I} \mu \mathrm{S} 3.0$ microfocus sealed tube (Mo K $\alpha$, $\lambda=0.71073 \AA$ )

Theta range for data collection

Index ranges

Reflections collected

Independent reflections

Absorption correction

Max. and min. transmission

Structure solution technique

Structure solution program

Refinement method

Refinement program

Function minimized

Data / restraints / parameters

Goodness-of-fit on $\mathrm{F}^{2}$

Final $\mathrm{R}$ indices

Largest diff. peak and hole
2.37 to $36.37^{\circ}$

$-11<=\mathrm{h}<=11,-21<=\mathrm{k}<=21,-38<=\mathrm{l}<=38$

133232

$9887[\mathrm{R}($ int $)=0.0367]$

Multi-Scan

0.8120 and 0.1580

direct methods

SHELXT 2014/5 (Sheldrick, 2014)

Full-matrix least-squares on $\mathrm{F}^{2}$

SHELXL-2017/1 (Sheldrick, 2017)

$\Sigma \mathrm{w}\left(\mathrm{F}_{\mathrm{o}}^{2}-\mathrm{F}_{\mathrm{c}}{ }^{2}\right)^{2}$

9887 / 9 / 239

1.244

9735 data; $\mathrm{I}>2 \sigma(\mathrm{I}) \mathrm{R} 1=0.0178, \mathrm{wR} 2=0.0424$

all data $\quad \mathrm{R} 1=0.0181, \mathrm{wR} 2=0.0425$

0.882 and $-0.850 \mathrm{e}^{-3}$ 
Table S19. Atomic coordinates and equivalent isotropic atomic displacement parameters $\left(\AA^{2}\right)$ for $\mathrm{Ca}_{2}\left(\mathrm{CH}_{3} \mathrm{SO}_{2}\right)_{2}\left(\mathrm{CH}_{3} \mathrm{SO}_{3}\right)\left(\mathrm{I}_{3}\right) \cdot\left(\mathrm{H}_{2} \mathrm{O}\right)_{3}$.

$\mathrm{U}(\mathrm{eq})$ is defined as one third of the trace of the orthogonalized $\mathrm{U}_{\mathrm{ij}}$ tensor.

\begin{tabular}{lllll} 
& \multicolumn{1}{c}{$\mathrm{x} / \mathrm{a}$} & \multicolumn{1}{c}{$\mathrm{y} / \mathrm{b}$} & \multicolumn{1}{c}{$\mathrm{z} / \mathrm{c}$} & \multicolumn{1}{c}{$\mathrm{U}(\mathrm{eq})$} \\
$\mathrm{Ca} 1$ & $0.36393(3)$ & $0.86685(2)$ & $0.69699(2)$ & $0.00947(4)$ \\
$\mathrm{Ca} 04$ & $0.63212(3)$ & $0.33726(2)$ & $0.72942(2)$ & $0.00860(3)$ \\
$\mathrm{S} 1$ & $0.12342(4)$ & $0.31870(2)$ & $0.67232(2)$ & $0.01153(5)$ \\
$\mathrm{S} 2$ & $0.63026(4)$ & $0.62346(2)$ & $0.69781(2)$ & $0.01137(5)$ \\
S3 & $0.85675(4)$ & $0.90217(2)$ & $0.64040(2)$ & $0.01204(5)$ \\
O1 & $0.29630(12)$ & $0.31257(8)$ & $0.71596(4)$ & $0.01340(15)$ \\
O2 & $0.95590(13)$ & $0.35921(8)$ & $0.70727(4)$ & $0.01442(15)$ \\
O1W & $0.34757(16)$ & $0.84078(10)$ & $0.59604(5)$ & $0.0217(2)$ \\
O3 & $0.59567(16)$ & $0.51328(7)$ & $0.70957(5)$ & $0.01861(18)$ \\
O2W & $0.36216(17)$ & $0.05087(9)$ & $0.71999(6)$ & $0.0226(2)$ \\
O4 & $0.79315(14)$ & $0.66580(7)$ & $0.73387(4)$ & $0.01441(15)$ \\
O3W & $0.60823(17)$ & $0.27070(10)$ & $0.63298(5)$ & $0.0215(2)$ \\
O5 & $0.45239(14)$ & $0.68509(8)$ & $0.70296(5)$ & $0.01628(16)$ \\
O6 & $0.03215(13)$ & $0.87518(9)$ & $0.67997(4)$ & $0.01558(16)$ \\
O7 & $0.68654(13)$ & $0.89829(8)$ & $0.68033(4)$ & $0.01423(15)$ \\
C1 & $0.1813(2)$ & $0.43268(12)$ & $0.63171(6)$ & $0.0180(2)$ \\
C2 & $0.6974(2)$ & $0.63099(12)$ & $0.62619(6)$ & $0.0221(3)$ \\
C3 & $0.8853(2)$ & $0.03940(12)$ & $0.62859(7)$ & $0.0237(3)$ \\
I1B & $0.3395(3)$ & $0.1051(2)$ & $0.52467(11)$ & $0.0255(4)$ \\
I2B & $0.0276(5)$ & $0.2612(3)$ & $0.50943(11)$ & $0.0119(3)$ \\
I3B & $0.7114(3)$ & $0.4149(2)$ & $0.50544(7)$ & $0.0140(3)$ \\
I1 & $0.34539(16)$ & $0.10975(10)$ & $0.52235(4)$ & $0.01730(15)$ \\
I2 & $0.0218(3)$ & $0.25794(16)$ & $0.50981(7)$ & $0.0170(3)$ \\
I3 & $0.7069(2)$ & $0.40947(15)$ & $0.50379(5)$ & $0.0248(3)$
\end{tabular}

Table S20. Bond lengths $(\AA)$ for $\mathrm{Ca}_{2}\left(\mathrm{CH}_{3} \mathrm{SO}_{2}\right)_{2}\left(\mathrm{CH}_{3} \mathrm{SO}_{3}\right)\left(\mathrm{I}_{3}\right) \cdot\left(\mathrm{H}_{2} \mathrm{O}\right)_{3}$.

$\begin{array}{llll}\mathrm{Ca} 1-\mathrm{O} 6 & 2.2993(9) & \mathrm{Ca} 1-\mathrm{O} 7 & 2.3018(9) \\ \mathrm{Ca} 1-\mathrm{O} 1 \mathrm{~W} & 2.3736(11) & \mathrm{Ca} 1-\mathrm{O} 5 & 2.3945(10) \\ \mathrm{Ca} 1-\mathrm{O} 2 \mathrm{~W} & 2.4028(11) & \mathrm{Ca} 1-\mathrm{O} 1 & 2.4490(9) \\ \mathrm{Ca} 1-\mathrm{O} 2 & 2.5058(10) & \mathrm{Ca} 1-\mathrm{S} 1 & 3.1037(4) \\ \mathrm{Ca} 1-\mathrm{Ca} 04 & 3.8152(3) & \mathrm{Ca} 1-\mathrm{Ca} 04 & 3.8994(3) \\ \mathrm{Ca} 04-\mathrm{O} 3 & 2.2991(10) & \mathrm{Ca} 04-\mathrm{O} 2 & 2.3224(9) \\ \mathrm{Ca} 04-\mathrm{O} 1 & 2.3363(9) & \mathrm{Ca} 04-\mathrm{O} 4 & 2.3916(9)\end{array}$




$\begin{array}{llll}\mathrm{Ca} 04-\mathrm{O} 3 \mathrm{~W} & 2.4006(11) & \mathrm{Ca} 04-\mathrm{O} 6 & 2.4841(10) \\ \mathrm{Ca} 04-\mathrm{O} 7 & 2.5200(10) & \mathrm{Ca} 04-\mathrm{S} 3 & 3.1415(4) \\ \mathrm{S} 1-\mathrm{O} 2 & 1.5280(10) & \mathrm{S} 1-\mathrm{O} 1 & 1.5317(9) \\ \mathrm{S} 1-\mathrm{C} 1 & 1.7864(14) & \mathrm{S} 2-\mathrm{O} 3 & 1.4504(10) \\ \mathrm{S} 2-\mathrm{O} 5 & 1.4615(10) & \mathrm{S} 2-\mathrm{O} 4 & 1.4722(10) \\ \mathrm{S} 2-\mathrm{C} 2 & 1.7532(15) & \mathrm{S} 3-\mathrm{O} 6 & 1.5244(10) \\ \mathrm{S} 3-\mathrm{O} 7 & 1.5265(10) & \mathrm{S} 3-\mathrm{C} 3 & 1.7804(15) \\ \mathrm{I} 1 \mathrm{~B}-\mathrm{I} 2 \mathrm{~B} & 2.932(3) & \mathrm{I} 2 \mathrm{~B}-\mathrm{I} 3 \mathrm{~B} & 2.923(3) \\ \mathrm{I} 1-\mathrm{I} 2 & 2.9208(19) & \mathrm{I} 2-\mathrm{I} 3 & 2.897(2)\end{array}$

Table S21. Bond angles ( ${ }^{\circ}$ ) for $\mathrm{Ca}_{2}\left(\mathrm{CH}_{3} \mathrm{SO}_{2}\right)_{2}\left(\mathrm{CH}_{3} \mathrm{SO}_{3}\right)\left(\mathrm{I}_{3}\right) \cdot\left(\mathrm{H}_{2} \mathrm{O}\right)_{3}$.

$\begin{array}{llll}\text { O6-Ca1-O7 } & 156.58(4) & \text { O6-Ca1-O1W } & 80.30(4) \\ \text { O7-Ca1-O1W } & 82.05(4) & \text { O6-Ca1-O5 } & 107.57(4) \\ \text { O7-Ca1-O5 } & 86.21(4) & \text { O1W-Ca1-O5 } & 85.61(4) \\ \text { O6-Ca1-O2W } & 88.79(4) & \text { O7-Ca1-O2W } & 83.25(4) \\ \text { O1W-Ca1-O2W } & 110.90(5) & \text { O5-Ca1-O2W } & 158.84(4) \\ \text { O6-Ca1-O1 } & 71.21(3) & \text { O7-Ca1-O1 } & 131.26(3) \\ \text { O1W-Ca1-O1 } & 141.06(4) & \text { O5-Ca1-O1 } & 78.55(4) \\ \text { O2W-Ca1-O1 } & 94.79(4) & \text { O6-Ca1-O2 } & 127.10(3) \\ \text { O7-Ca1-O2 } & 73.43(3) & \text { O1W-Ca1-O2 } & 151.32(4) \\ \text { O5-Ca1-O2 } & 78.26(4) & \text { O2W-Ca1-O2 } & 81.16(4) \\ \text { O1-Ca1-O2 } & 58.27(3) & \text { O6-Ca1-S1 } & 99.42(3) \\ \text { O7-Ca1-S1 } & 102.33(3) & \text { O1W-Ca1-S1 } & 160.54(3) \\ \text { O5-Ca1-S1 } & 75.88(3) & \text { O2W-Ca1-S1 } & 88.51(3) \\ \text { O1-Ca1-S1 } & 29.09(2) & \text { O2-Ca1-S1 } & 29.21(2) \\ \text { O6-Ca1-Ca04 } & 162.89(3) & \text { O7-Ca1-Ca04 } & 39.75(2) \\ \text { O1W-Ca1-Ca04 } & 115.76(3) & \text { O5-Ca1-Ca04 } & 69.84(2) \\ \text { O2W-Ca1-Ca04 } & 90.53(3) & \text { O1-Ca1-Ca04 } & 91.82(2) \\ \text { O2-Ca1-Ca04 } & 36.14(2) & \text { S1-Ca1-Ca04 } & 63.469(8) \\ \text { O6-Ca1-Ca04 } & 37.00(2) & \text { O7-Ca1-Ca04 } & 163.21(3) \\ \text { O1W-Ca1-Ca04 } & 114.68(3) & \text { O5-Ca1-Ca04 } & 96.06(3) \\ \text { O2W-Ca1-Ca04 } & 89.03(3) & \text { O1-Ca1-Ca04 } & 34.48(2) \\ \text { O2-Ca1-Ca04 } & 90.70(2) & \text { S1-Ca1-Ca04 } & 62.440(8) \\ \text { Ca04-Ca1-Ca04 } & 125.900(9) & \text { O3-Ca04-O2 } & 86.28(4) \\ \text { O3-Ca04-O1 } & 90.33(4) & \text { O2-Ca04-O1 } & 159.44(3) \\ \text { O3-Ca04-O4 } & 168.80(4) & \text { O2-Ca04-O4 } & 89.80(4) \\ \text { O1-Ca04-O4 } & 96.94(4) & \text { O3-Ca04-O3W } & 98.81(4)\end{array}$




\begin{tabular}{|c|c|c|c|}
\hline $\mathrm{O} 2-\mathrm{Ca} 04-\mathrm{O} 3 \mathrm{~W}$ & $82.05(4)$ & $\mathrm{O} 1-\mathrm{Ca} 04-\mathrm{O} 3 \mathrm{~W}$ & $78.45(4)$ \\
\hline O4-Ca04-O3W & $91.02(4)$ & O3-Ca04-O6 & $86.11(4)$ \\
\hline O2-Ca04-O6 & $129.83(3)$ & O1-Ca04-O6 & $69.99(3)$ \\
\hline O4-Ca04-O6 & $88.38(4)$ & $\mathrm{O} 3 \mathrm{~W}-\mathrm{Ca} 04-\mathrm{O} 6$ & $148.11(4)$ \\
\hline O3-Ca04-O7 & $85.01(4)$ & O2-Ca04-O7 & $72.82(3)$ \\
\hline O1-Ca04-O7 & $127.10(3)$ & O4-Ca04-O7 & $83.81(3)$ \\
\hline O3W-Ca04-O7 & $154.31(4)$ & O6-Ca04-O7 & $57.13(3)$ \\
\hline $\mathrm{O} 3-\mathrm{Ca} 04-\mathrm{S} 3$ & $86.32(3)$ & $\mathrm{O} 2-\mathrm{Ca} 04-\mathrm{S} 3$ & $101.51(3)$ \\
\hline O1-Ca04-S3 & $98.49(2)$ & $\mathrm{O} 4-\mathrm{Ca} 04-\mathrm{S} 3$ & $84.19(3)$ \\
\hline $\mathrm{O} 3 \mathrm{~W}-\mathrm{Ca} 04-\mathrm{S} 3$ & $173.99(3)$ & O6-Ca04-S3 & $28.50(2)$ \\
\hline O7-Ca04-S3 & $28.69(2)$ & $\mathrm{O} 3-\mathrm{Ca} 04-\mathrm{Ca} 1$ & $94.76(3)$ \\
\hline O2-Ca04-Ca1 & $39.52(2)$ & O1-Ca04-Ca1 & $160.98(2)$ \\
\hline O4-Ca04-Ca1 & $75.68(2)$ & O3W-Ca04-Ca1 & $118.65(3)$ \\
\hline O6-Ca04-Ca1 & $92.05(2)$ & O7-Ca04-Ca1 & $35.74(2)$ \\
\hline S3-Ca04-Ca1 & $63.672(7)$ & O3-Ca04-Ca1 & $84.53(3)$ \\
\hline O2-Ca04-Ca1 & $161.83(3)$ & O1-Ca04-Ca1 & $36.40(2)$ \\
\hline O4-Ca04-Ca1 & $96.26(3)$ & O3W-Ca04-Ca1 & $114.84(3)$ \\
\hline O6-Ca04-Ca1 & $33.85(2)$ & O7-Ca04-Ca1 & $90.76(2)$ \\
\hline S3-Ca04-Ca1 & $62.300(8)$ & Ca1-Ca04-Ca1 & $125.900(9)$ \\
\hline O2-S1-O1 & $104.11(5)$ & $\mathrm{O} 2-\mathrm{S} 1-\mathrm{C} 1$ & $101.44(6)$ \\
\hline $\mathrm{O} 1-\mathrm{S} 1-\mathrm{C} 1$ & $102.07(6)$ & $\mathrm{O} 2-\mathrm{S} 1-\mathrm{Ca} 1$ & $53.15(4)$ \\
\hline O1-S1-Ca1 & $51.01(4)$ & C1-S1-Ca1 & $111.23(5)$ \\
\hline O3-S2-O5 & $111.03(6)$ & $\mathrm{O} 3-\mathrm{S} 2-\mathrm{O} 4$ & $111.89(6)$ \\
\hline O5-S2-O4 & $111.98(6)$ & $\mathrm{O} 3-\mathrm{S} 2-\mathrm{C} 2$ & $106.55(7)$ \\
\hline $\mathrm{O} 5-\mathrm{S} 2-\mathrm{C} 2$ & $107.72(7)$ & $\mathrm{O} 4-\mathrm{S} 2-\mathrm{C} 2$ & $107.36(7)$ \\
\hline O6-S3-O7 & $103.33(5)$ & O6-S3-C3 & $103.00(7)$ \\
\hline $\mathrm{O} 7-\mathrm{S} 3-\mathrm{C} 3$ & $102.63(7)$ & O6-S3-Ca04 & $51.04(4)$ \\
\hline O7-S3-Ca04 & $52.42(4)$ & $\mathrm{C} 3-\mathrm{S} 3-\mathrm{Ca} 04$ & $114.15(6)$ \\
\hline $\mathrm{S} 1-\mathrm{O} 1-\mathrm{Ca} 04$ & $144.61(6)$ & S1-O1-Ca1 & $99.90(4)$ \\
\hline $\mathrm{Ca} 04-\mathrm{O} 1-\mathrm{Ca} 1$ & $109.13(4)$ & S1-O2-Ca04 & $145.97(6)$ \\
\hline S1-O2-Ca1 & $97.65(4)$ & $\mathrm{Ca} 04-\mathrm{O} 2-\mathrm{Ca} 1$ & $104.34(4)$ \\
\hline $\mathrm{S} 2-\mathrm{O} 3-\mathrm{Ca} 04$ & $164.33(7)$ & $\mathrm{S} 2-\mathrm{O} 4-\mathrm{Ca} 04$ & $133.18(6)$ \\
\hline S2-O5-Ca1 & $136.46(6)$ & S3-O6-Ca1 & $150.06(6)$ \\
\hline S3-O6-Ca04 & $100.45(4)$ & Ca1-O6-Ca04 & $109.15(4)$ \\
\hline $\mathrm{S} 3-\mathrm{O} 7-\mathrm{Ca} 1$ & $151.30(6)$ & S3-O7-Ca04 & $98.88(4)$ \\
\hline Ca1-O7-Ca04 & $104.51(4)$ & I3B-I2B-I1B & $174.78(13)$ \\
\hline I3-I2-I1 & $176.75(7)$ & & \\
\hline
\end{tabular}


Table S22. Torsion angles ( $\left.{ }^{\circ}\right)$ for $\mathrm{Ca}_{2}\left(\mathrm{CH}_{3} \mathrm{SO}_{2}\right)_{2}\left(\mathrm{CH}_{3} \mathrm{SO}_{3}\right)\left(\mathrm{I}_{3}\right) \cdot\left(\mathrm{H}_{2} \mathrm{O}\right)_{3}$.

$\begin{array}{lrll}\text { O2-S1-O1-Ca04 } & -142.67(10) & \text { C1-S1-O1-Ca04 } & -37.43(12) \\ \text { Ca1-S1-O1-Ca04 } & -145.09(13) & \text { O2-S1-O1-Ca1 } & 2.41(6) \\ \text { C1-S1-O1-Ca1 } & 107.66(6) & \text { O1-S1-O2-Ca04 } & -132.55(10) \\ \text { C1-S1-O2-Ca04 } & 121.73(11) & \text { Ca1-S1-O2-Ca04 } & -130.21(12) \\ \text { O1-S1-O2-Ca1 } & -2.35(6) & \text { C1-S1-O2-Ca1 } & -108.06(5) \\ \text { O5-S2-O3-Ca04 } & -166.5(3) & \text { O4-S2-O3-Ca04 } & -40.5(3) \\ \text { C2-S2-O3-Ca04 } & 76.5(3) & \text { O3-S2-O4-Ca04 } & -151.37(8) \\ \text { O5-S2-O4-Ca04 } & -25.97(10) & \text { C2-S2-O4-Ca04 } & 92.07(9) \\ \text { O3-S2-O5-Ca1 } & 176.28(8) & \text { O4-S2-O5-Ca1 } & 50.41(11) \\ \text { C2-S2-O5-Ca1 } & -67.41(11) & \text { O7-S3-O6-Ca1 } & -167.23(13) \\ \text { C3-S3-O6-Ca1 } & -60.66(15) & \text { Ca04-S3-O6-Ca1 } & -171.35(16) \\ \text { O7-S3-O6-Ca04 } & 4.11(6) & \text { C3-S3-O6-Ca04 } & 110.69(7) \\ \text { O6-S3-O7-Ca1 } & -148.50(12) & \text { C3-S3-O7-Ca1 } & 104.64(13) \\ \text { Ca04-S3-O7-Ca1 } & -144.47(15) & \text { O6-S3-O7-Ca04 } & -4.04(6) \\ \text { C3-S3-O7-Ca04 } & -110.89(6) & & \end{array}$

Table S23. Anisotropic atomic displacement parameters $\left(\AA^{2}\right)$ for $\mathrm{Ca}_{2}\left(\mathrm{CH}_{3} \mathrm{SO}_{2}\right)_{2}\left(\mathrm{CH}_{3} \mathrm{SO}_{3}\right)\left(\mathrm{I}_{3}\right) \cdot\left(\mathrm{H}_{2} \mathrm{O}\right)_{3}$.

The anisotropic atomic displacement factor exponent takes the form: $-2 \pi^{2}\left[h^{2} a^{* 2} U_{11}+\ldots+2 h k^{*} b^{*} U_{12}\right]$

$\begin{array}{llllll}\mathrm{U}_{11} & \mathrm{U}_{22} & \mathrm{U}_{33} & \mathrm{U}_{23} & \mathrm{U}_{13} & \mathrm{U}_{12}\end{array}$

$\begin{array}{lrrrrrr}\text { Ca1 } & 0.00602(7) & 0.01137(8) & 0.01102(8) & 0.00075(6) & 0.00025(6) & 0.00052(6) \\ \text { Ca04 } & 0.00612(7) & 0.00936(8) & 0.01030(8) & 0.00012(6) & 0.00019(6) & 0.00021(6) \\ \text { S1 } & 0.00773(9) & 0.01656(12) & 0.01027(10) & -0.00103(9) & -0.00010(8) & -0.00075(8) \\ \text { S2 } & 0.01183(10) & 0.00845(10) & 0.01364(11) & -0.00042(8) & -0.00162(9) & 0.00075(8) \\ \text { S3 } & 0.00842(10) & 0.01708(12) & 0.01060(10) & 0.00217(9) & 0.00023(8) & 0.00055(9) \\ \text { O1 } & 0.0070(3) & 0.0216(4) & 0.0115(3) & -0.0001(3) & -0.0005(3) & -0.0004(3) \\ \text { O2 } & 0.0071(3) & 0.0223(4) & 0.0141(4) & -0.0002(3) & 0.0019(3) & -0.0002(3) \\ \text { O1W } & 0.0191(4) & 0.0332(6) & 0.0127(4) & -0.0018(4) & 0.0004(3) & -0.0034(4) \\ \text { O3 } & 0.0199(4) & 0.0086(3) & 0.0267(5) & 0.0017(3) & -0.0062(4) & -0.0009(3) \\ \text { O2W } & 0.0187(4) & 0.0145(4) & 0.0341(6) & -0.0048(4) & -0.0057(4) & 0.0022(3) \\ \text { O4 } & 0.0144(4) & 0.0113(3) & 0.0172(4) & -0.0015(3) & -0.0029(3) & -0.0005(3) \\ \text { O3W } & 0.0198(4) & 0.0304(5) & 0.0145(4) & -0.0063(4) & 0.0020(3) & -0.0021(4) \\ \text { O5 } & 0.0132(4) & 0.0128(4) & 0.0227(4) & -0.0005(3) & -0.0010(3) & 0.0030(3) \\ \text { O6 } & 0.0069(3) & 0.0248(4) & 0.0149(4) & 0.0045(3) & -0.0001(3) & 0.0021(3)\end{array}$




\begin{tabular}{lrrrrrr} 
& $\mathrm{U}_{11}$ & $\mathrm{U}_{22}$ & $\mathrm{U}_{33}$ & \multicolumn{1}{c}{$\mathrm{U}_{23}$} & \multicolumn{1}{c}{$\mathrm{U}_{13}$} & \multicolumn{1}{c}{$\mathrm{U}_{12}$} \\
$\mathrm{O} 7$ & $0.0069(3)$ & $0.0209(4)$ & $0.0151(4)$ & $0.0040(3)$ & $0.0016(3)$ & $-0.0005(3)$ \\
$\mathrm{C} 1$ & $0.0162(5)$ & $0.0223(6)$ & $0.0153(5)$ & $0.0037(4)$ & $0.0009(4)$ & $-0.0014(4)$ \\
$\mathrm{C} 2$ & $0.0291(7)$ & $0.0220(6)$ & $0.0154(5)$ & $-0.0037(4)$ & $0.0034(5)$ & $0.0005(5)$ \\
C3 & $0.0241(6)$ & $0.0200(6)$ & $0.0271(7)$ & $0.0097(5)$ & $0.0026(5)$ & $-0.0022(5)$ \\
I1B & $0.0211(4)$ & $0.0237(4)$ & $0.0323(7)$ & $0.0044(3)$ & $0.0053(3)$ & $0.0095(3)$ \\
I2B & $0.0140(4)$ & $0.0128(4)$ & $0.0091(4)$ & $-0.0020(3)$ & $0.0025(3)$ & $0.0002(3)$ \\
I3B & $0.0173(4)$ & $0.0147(3)$ & $0.0098(4)$ & $-0.00073(19)$ & $-0.0010(2)$ & $0.0049(2)$ \\
I1 & $0.01582(18)$ & $0.02157(19)$ & $0.0145(3)$ & $0.00012(13)$ & $0.00096(12)$ & $0.00363(15)$ \\
I2 & $0.0194(4)$ & $0.0181(4)$ & $0.0134(3)$ & $-0.00055(19)$ & $-0.0008(2)$ & $0.0009(2)$ \\
I3 & $0.0274(3)$ & $0.0220(3)$ & $0.0248(4)$ & $0.00155(18)$ & $-0.0009(2)$ & $0.00840(19)$
\end{tabular}

Table S24. Hydrogen atomic coordinates and isotropic atomic displacement parameters $\left(\AA^{2}\right)$ for $\mathrm{Ca}_{2}\left(\mathrm{CH}_{3} \mathrm{SO}_{2}\right)_{2}\left(\mathrm{CH}_{3} \mathrm{SO}_{3}\right)\left(\mathrm{I}_{3}\right) \cdot\left(\mathrm{H}_{2} \mathrm{O}\right)_{3}$.

\begin{tabular}{lllll} 
& \multicolumn{1}{c}{$\mathrm{x} / \mathrm{a}$} & \multicolumn{1}{c}{$\mathrm{y} / \mathrm{b}$} & \multicolumn{1}{c}{$\mathrm{z} / \mathrm{c}$} & \multicolumn{1}{c}{$\mathrm{U}(\mathrm{eq})$} \\
$\mathrm{H} 1 \mathrm{~A}$ & 1.2965 & 0.4186 & 0.6096 & 0.027 \\
$\mathrm{H} 1 \mathrm{~B}$ & 1.2081 & 0.4917 & 0.6579 & 0.027 \\
$\mathrm{H} 1 \mathrm{C}$ & 1.0710 & 0.4503 & 0.6054 & 0.027 \\
$\mathrm{H} 2 \mathrm{~A}$ & 0.5932 & 0.6016 & 0.6010 & 0.033 \\
$\mathrm{H} 2 \mathrm{~B}$ & 0.8176 & 0.5910 & 0.6216 & 0.033 \\
H2C & 0.7190 & 0.7046 & 0.6159 & 0.033 \\
H3A & 0.0043 & 1.0517 & 0.6078 & 0.036 \\
H3B & -0.1051 & 1.0760 & 0.6656 & 0.036 \\
H3C & -0.2273 & 1.0660 & 0.6058 & 0.036 \\
H1WA & $0.258(3)$ & $0.8128(19)$ & $0.5759(10)$ & 0.028 \\
H1WB & $0.423(3)$ & $0.861(2)$ & $0.5705(9)$ & 0.028 \\
H2WA & $0.467(3)$ & $1.0797(19)$ & $0.7333(11)$ & 0.028 \\
H2WB & $0.271(3)$ & $1.0880(18)$ & $0.7302(11)$ & 0.028 \\
H3WA & $0.536(3)$ & $0.2234(16)$ & $0.6202(11)$ & 0.028 \\
H3WB & $0.651(4)$ & $0.3016(18)$ & $0.6051(9)$ & 0.028
\end{tabular}


Table S25. Hydrogen bond distances $(\AA)$ and angles $\left(^{\circ}\right)$ for $\mathrm{Ca}_{2}\left(\mathrm{CH}_{3} \mathrm{SO}_{2}\right)_{2}\left(\mathrm{CH}_{3} \mathrm{SO}_{3}\right)\left(\mathrm{I}_{3}\right) \cdot\left(\mathrm{H}_{2} \mathrm{O}\right)_{3}$.

Donor-H Acceptor-H Donor-Acceptor Angle

\begin{tabular}{|c|c|c|c|}
\hline $\mathrm{O} 2 \mathrm{~W}-\mathrm{H} 2 \mathrm{WA}^{\cdots} \mathrm{O}^{-\mathrm{O}}$ & $0.852(16) 2.098(16)$ & $2.9468(15)$ & 174.(2) \\
\hline O1W-H1WB“'I1B' & $0.842(16) 2.846(17)$ & $3.679(4)$ & $170 .(2)$ \\
\hline O3W-H3WA'“I1B' & $0.829(16) 2.962(19)$ & $3.717(2)$ & 152.(2) \\
\hline O1W-H1WA“'I2B' & $0.838(16) 2.889(17)$ & $3.717(4)$ & $170 .(2)$ \\
\hline $\mathrm{O} 2 \mathrm{~W}-\mathrm{H} 2 \mathrm{WB} \cdots \mathrm{O} 5$ & $0.826(16) 2.555(18)$ & $3.3391(16)$ & 159.(2) \\
\hline O3W-H3WB'“'I3B' & $0.824(16) 2.780(17)$ & $3.5899(19)$ & 168.(2) \\
\hline $\mathrm{C} 2-\mathrm{H} 2 \mathrm{~A}^{\cdots \cdots} \mathrm{I} 3 \mathrm{~B}^{\prime}$ & 0.98 & $4.110(3)$ & 158.5 \\
\hline $\mathrm{C} 2-\mathrm{H} 2 \mathrm{C}^{\cdots} \mathrm{S} 3$ & 0.98 & $3.6318(16)$ & 151.9 \\
\hline C3-H3A' $\cdots 11 B^{\prime}$ & 0.98 & $4.118(4)$ & 170.5 \\
\hline
\end{tabular}

